\title{
Asymmetry in Exchange Rate Pass-Through to Consumer Prices: New Perspective from Sub-Saharan African Countries
}

\author{
Diby François Kassi ${ }^{1, *}$, Dilesha Nawadali Rathnayake ${ }^{1}\left(\mathbb{D}\right.$, Akadje Jean Roland Edjoukou ${ }^{2}$, \\ Yobouet Thierry Gnangoin ${ }^{3}$, Pierre Axel Louembe ${ }^{2}$, Ning Ding ${ }^{1}$ and Gang Sun ${ }^{1}$ \\ 1 School of Finance, Dongbei University of Finance and Economics, 217 Jianshan Street, Dalian 116023, China; \\ dileshausj@yahoo.com (D.N.R.); dingning610@dufe.edu.cn (N.D.); gangsun@dufe.edu.cn (G.S.) \\ 2 School of Accounting, Dongbei University of Finance and Economics, 217 Jianshan Street, Dalian 116023, \\ China; edjoukou2015@yahoo.fr (A.J.R.E.); louembepierre@yahoo.fr (P.A.L.) \\ 3 School of Economics, Dongbei University of Finance and Economics, 217 Jianshan Street, Dalian 116023, \\ China; thierrybienvenu2010@yahoo.fr \\ * Correspondence: kassidiby17@gmail.com or kassidibyfnk1@yahoo.fr; Tel.: +86-130-0941-2172
}

Received: 18 October 2018; Accepted: 3 January 2019; Published: 11 January 2019

\begin{abstract}
This paper examines the asymmetrical relationship between exchange rate and consumer prices in 40 sub-Saharan African (SSA) countries from 1990Q1 to 2017Q4. We estimate the exchange rate pass-through (ERPT) to consumer prices for each country by using the nonlinear autoregressive distributed lag (NARDL) framework and dynamic panel techniques robust to cross-sectional dependence. First, our findings suggest an asymmetrical ERPT in the SSA region during the short term, whereas there are mixed results across subregions in the long term. Second, the results of the panel analysis suggest incomplete and significant ERPT to consumer prices in the entire SSA region, which is higher during depreciation of the local currency than after appreciation in the short-term, especially in the CFA Franc zone. Third, we find nonlinear ERPT with respect to the size of the exchange rate. Finally, we find that pass-through is higher in countries with fixed exchange rate regimes (CFA franc zone) in a low inflationary environment than in countries with floating exchange rate regimes and high inflation levels. Pass-through is greater during large exchange rate changes than after small changes. Therefore, the policy implication is to consider these asymmetries and nonlinearities to improve monetary policy's credibility, enhance trade liberalization, and promote competitive market structures in the SSA region.
\end{abstract}

Keywords: asymmetry; exchange rate pass-through; NARDL; inflation; sub-Saharan Africa

\section{Introduction}

Interest in exchange rate pass-through analysis is justified by the need to understand how economic cycles, trade imbalances, and especially exchange rate changes affect domestic prices and monetary policy. Exchange rate pass-through (ERPT) denotes the degree to which exchange rate changes are transferred into prices in a particular country. Several studies have analyzed the ERPT to import and consumer prices, initially in developed countries, suggesting that it has fallen since the 1990s. Taylor (2000) found that ERPT declined under a low inflationary environment in the US during the 1990s, and this hypothesis has been largely validated by other studies in advanced nations, such as Campa and Goldberg (2002), Choudhri et al. (2005), Campa and Goldberg (2005), and Takhtamanova (2010). Most of these studies ignored the likely asymmetrical ERPT to prices (inflation) underlined in the theoretical models of pricing to market, where foreign exporters adjust the prices in the importing country in response to the size and direction of exchange 
rate movements. Accordingly, recent studies consider nonlinearities and asymmetries in their investigation of the relationship between exchange rates and domestic prices in developed and emerging economies (Brun-Aguerre et al. 2012, 2016; Choudhri and Hakura 2015; Yanamandra 2015; Baharumshah et al. 2017, among others). In fact, these studies revealed an asymmetrical and nonlinear ERPT to import prices as well as a more complete and higher pass-through during exchange rate depreciations than appreciations in the long term.

Examining the relationship between exchange rate and prices is an important step for sub-Saharan African (SSA) countries to secure their economies against structural shocks during global trade. By the early 2000s, several SSA countries had benefited from a stable and low inflation level with sustained growth. However, the policy framework presents some weaknesses because of the limited role of the exchange rate used as a nominal anchor. One common feature with SSA countries that distinguishes them from other regions is a lack of credibility of monetary policy. At earlier stages of credibility, the trade-off between output and inflation is more severe. Most SSA countries focus on inflation-targeting policies, to the detriment of the anchoring role of the exchange rate. Most of the SSA countries, i.e., the CFA franc region (West African Economic and Monetary Union (WAEMU) and Economic and Monetary Community of Central Africa (CEMAC)), have pegged their currency to the euro, resulting in low inflation levels in this region compared with other SSA countries. The importance of exchange rate dynamics and their likely asymmetrical effects on a country's macroeconomic environment have been underlined in recent studies (Brun-Aguerre et al. 2016; Bui 2018; Conrad and Jagessar 2018; Kassi et al. 2018; Usman and Elsalih 2018).

Concerning SSA countries, the literature on ERPT is limited and based on some specific countries rather than the whole region (Bhundia 2002; Mwase 2006; Karoro et al. 2009; Maka 2013; Jooste and Jhaveri 2014; Bada et al. 2016). However, Akofio-Sowah (2009) examined the relationship between ERPT and monetary regime in 15 SSA countries during the period 1980-2005. He concluded that the pass-through was lower in countries under a low inflationary environment. The International Monetary Fund's (IMF) working paper of Razafimahefa (International Monetary Fund 2012) extended the ERPT analysis to 34 SSA countries on a quarterly time series from 1980 to 2005 using a vector autoregression (VAR) framework. The author suggested that there was a declining pass-through in the 1990s due to macroeconomic reforms. Most of these studies are at the country level and neglect the asymmetry and nonlinearity assumption between exchange rate changes and domestic prices in the SSA countries, as well as cross-sectional dependence across countries, which may lead to biased results.

Accordingly, our study examines the asymmetry and nonlinearities of ERPT to consumer prices from 1990Q1 to 2017Q4 for 40 SSA countries split into two groups: the CFA franc zone, with a fixed exchange rate regime (WAEMU and CEMAC, 14 countries), and the other SSA countries, with a floating exchange rate regime (26 countries). Then, we also consider cross-sectional dependence between countries, which may arise from unobserved common factors. In particular, we conduct tests on the following hypotheses: Is there a symmetrical ERPT to consumer prices during depreciations and appreciations? Is there a nonsignificant ERPT in the long term? Is there evidence of a complete ERPT in the SSA countries? Does the size of the exchange rate matter in the likely asymmetrical ERPT?

First, we estimate the ERPT for each country by employing the nonlinear autoregressive distributed lag (NARDL) framework of Shin et al. (2014) and the general to specific approach with a maximum lag length of 4, dropping all insignificant variables in the regression. The results reject the hypothesis of a symmetrical ERPT for most of the SSA countries (30 cases in the long term and 24 out of 40 in the short term). We also find an incomplete ERPT except for six cases where the evidence of a complete ERPT are only found for depreciations in Angola and Kenya and one for appreciation in Liberia. Then, we find an asymmetrical and complete ERPT in Ghana and Lesotho over the long term and one case in the short term for depreciation in Mozambique. In addition, we unveil many cases of significant ERPT over the short and long term. Moreover, the dynamic multipliers also confirm the asymmetrical pattern of exchange rate to local prices in most of the cases. 
Second, the cross-sectional dependence tests confirm the existence of cross-sectionally correlated residuals between the panel units. Third, we employ the feasible generalized least squares (FGLS) estimator of Parks (1967) and the pooled ordinary least squares (OLS) regression with Driscoll and Kraay (1998) standard errors, which are consistent to heteroscedasticity and cross-sectionally correlated residuals. These panel estimators validate both the short- and long-term asymmetrical ERPT for the CFA franc zone and the short-term asymmetrical ERPT for the other SSA countries and the entire region. Our findings of an incomplete and non-zero ERPT are robust across the SSA countries under the cross-sectional dependence analysis where depreciations are strongly passed through to consumer prices more than appreciations over the short and long term, especially in the CFA Franc zone. The ERPT is higher in the CFA franc zone, which has a lower inflationary environment and less price volatility than the other SSA countries, in contrast to Taylor (2000) hypothesis. Furthermore, the pass-through coefficients become lower under the cross-sectional dependence analysis and higher in the long term than the short term. Finally, we find an asymmetrical ERPT with regard to the size of the exchange rate. The pass-through is higher after large exchange rate changes than small changes. In particular, the ERPT during large depreciations of the local currency is greater than that for large appreciations, whereas the ERPT for small appreciations is higher than that for small depreciations.

Our contribution to the ERPT literature in the SSA region is threefold: First, we extend the analysis to 40 SSA countries using both per-country and dynamic panel analysis, allowing for asymmetry and nonlinearities. Second, we consider the cross-sectional dependence analysis between countries in the estimation of ERPT. Finally, we examine the pass-through with regard to the size of the exchange rate change. Our findings reveal a lack of credibility of monetary policy in the SSA region and evidence of speculative behavior from foreign producers. This raises concerns about the deterioration of consumer welfare in the destination market following the response of local prices, thereby hindering the monetary policy of inflation targeting and export competitiveness. The asymmetrical ERPT also reflects downward price rigidities and weak market competition in many SSA countries. Therefore, these countries could benefit from trade liberalization and competitive market structures.

The remainder of this paper is structured as follows: Section 2 presents the literature on ERPT; Section 3 describes the data and the methodology used in this study; Section 4 presents the findings and discussion; and Section 5 provides the conclusion and policy implications.

\section{Literature Review}

\subsection{Theoretical Review}

The asymmetrical behavior of exchange rate changes in prices is often underlined in the microeconomic context of pricing to market theory, by stating that foreign firms are prone to adjust their markups in the importing country in response to exchange rate changes (Dornbusch 1985; Krugman 1986). This likely asymmetrical pattern of exchange rate is largely explained in the market share model (Marston 1990), where foreign exporters tend to pass through the appreciation of the importer's currency in order to enhance their market share, while absorbing the depreciation to maintain their profits. Thus, the exchange rate pass-through (ERPT) is higher during appreciation of the importer's currency than depreciation, similar to the technology switching model (see Ware and Winter 1988). Conversely, the capacity constraints model of Knetter (1994) posits that foreign firms are inclined to pass through depreciation of the importer's currency and absorb appreciation because they operate at full capacity and may not be able to contain huge demand when the importer's currency appreciates. Pollard and Coughlin (2004) demonstrate that asymmetry may arise from the pricing strategy of a foreign firm in response to the size of the exchange rate change. Thus, under the hypothesis that foreign exporters set invoice prices in their own currency, they have less incentive to adjust prices following small changes in the exchange rate, so that the prices the importer faces fully reflects the exchange rate changes. This strategy is termed producer currency pricing (PCP), where there is a complete ERPT. On the other hand, prices are not sensitive to small changes in the 
exchange rate when exporters set invoice prices in the importer's currency, which is consistent with the local currency pricing (LCP) strategy. In this case, the ERPT is zero and may increase if prices adjust to large exchange rate changes. Ben Cheikh (2012) provided a theoretical background for an asymmetrical ERPT in line with the production switching and market share models, in which the ERPT is greater after an appreciation of the importer's currency than during depreciation. He also justified the asymmetrical pass-through by the existence of menu costs, implying a larger ERPT in the wake of large changes in exchange rate than with small changes. Other recent studies have underlined the importance of macroeconomic factors (inflationary environment, the size of a country, exchange rate volatility, exchange rate regime, monetary policy, etc.) that influence the relationship between exchange rates and consumer prices (An and Wang 2011; Ben Cheikh 2012; Yellen 2015; Savoie-Chabot and Khan 2015, among others). For instance, An and Wang (2011) explained that the size of a country as measured by gross domestic product (GDP) is inversely associated with the degree of ERPT. In fact, foreign producers are prone to preserve their market shares in a large economy. Thus, they have more incentive to absorb exchange rate fluctuations in the currency of a large importing country by adjusting their markups.

In addition, a large economy tends to reduce its imports in response to the inflationary environment caused by a depreciation of its currency. The decreasing demand will lower the world prices of its imports and the ERPT if the country is a major importer in the world market of certain products. Moreover, Savoie-Chabot and Khan (2015) pointed out that monetary policy influences the extent of ERPT to consumer prices. The inflationary environment caused by depreciation of the local currency can be mitigated by an effective monetary policy targeting a low level of inflation, thereby reducing the degree of ERPT to consumer prices in the long term.

Most recent studies have focused on empirical analysis, giving support to the theoretical background of the likely asymmetrical relationship between exchange rate changes and consumer prices.

\subsection{Empirical Review}

Most of the studies on the relationship between exchange rate changes and local prices initially suggested a symmetrical and declining exchange rate pass-through (ERPT) to prices over the years, especially in developed countries (Taylor 2000; Olivei 2002; Campa and Goldberg 2005; among others). For instance, Otani et al. (2003) found a lower ERPT to import prices for Japanese industries in the 1990s using monthly data from 1978 to 2002 on both overall and disaggregate import prices. Likewise, Takhtamanova (2010) validated Taylor (2000) hypothesis of a declining ERPT under a low inflationary regime during the 1990s in 14 developed nations. This hypothesis has been supported by several other studies (Choudhri et al. 2005; Frankel et al. 2011; Ozkan and Erden 2015). Several empirical studies concluded that the pass-through to prices was incomplete and smaller in developed nations, lying between 0 and 1, than in developing countries (Goldberg and Knetter 1996; Berner 2010; Bussière et al. 2014, among others). Berner (2010) studied the ERPT to import unit value in Germany using monthly data from 1988 to 2008. He found an incomplete and nonlinear ERPT, which was higher during depreciations of the euro than appreciations, differing across trading partners.

Investigation of the relationship between exchange rates and prices has moved forward by allowing for asymmetric and nonlinear ERPT to price levels in developed and emerging countries (Delatte and Lòpez-Villavicencio 2012; Yanamandra 2015; Brun-Aguerre et al. 2016; Baharumshah et al. 2017; Kassi et al. 2018, among others). Brun-Aguerre et al. (2016) investigated the ERPT to import prices for an unbalanced panel data of 14 emerging markets and 19 developed markets from 1980Q1 to 2010Q4. Most of these studies employed the nonlinear autoregressive distributed lag (NARDL) framework of Shin et al. (2014) and revealed an asymmetrical ERPT in which exchange rate depreciations were passed through to prices more strongly than appreciations in the long term. Utku Özmen and Akçelik (2017) utilized micro data to investigate the impact of oil prices and exchange rate on retail motor fuel prices in Turkey from 1 January 2006 to 14 February 2014. They 
showed an asymmetrical response of motor fuel prices, which was intensified by exchange rate changes (oil price) in the case of positive (negative) cost shock. They also showed that the pass-through size was inversely associated with the level of positive cost shock, and concluded that the market structure was the major reason for this asymmetry. Additionally, Kassi et al. (2018) found an asymmetrical ERPT in developing and emerging Asian countries by using the NARDL framework on quarterly data from 1995Q1 to 2016Q4.

The research on ERPT in sub-Saharan African countries has been scarce, largely dominated by country-level studies. Bhundia (2002) analyzed the ERPT to consumer prices in South Africa and found a lower pass-through by using a vector autoregressive (VAR) framework on quarterly data from 1976Q2 to 2000Q3.

Mwase (2006) also showed a declining ERPT in Tanzania in the 1990s through a structural VAR model with a dataset from 1990 to 2005. He argued that the lower pass-through was in part attributed to the structural and macroeconomic reforms during the 1990s. Moreover, Akofio-Sowah (2009) investigated the relationship between exchange rate pass-through and the monetary regime in 12 emerging countries and 15 sub-Saharan African countries from 1980 to 2005. The author revealed that ERPT was lower in countries with low inflation levels, especially in the CFA franc zone and the common monetary area (CMA) than others. Another study, by Frimpong and Adam (2010), examined the ERPT to Ghanaian consumer prices with a VAR model on monthly data over 1990M1-2009M2 periods. They concluded that there was a declining and incomplete ERPT to prices, which was significant in the short term. In the IMF's working papers, Razafimahefa (International Monetary Fund 2012) gave an analysis covering 34 SSA countries, split into fixed regimes ( 23 countries) and flexible regimes (11 countries). The author analyzed ERPT and its determinants in these countries on quarterly data from 1985 to 2008, and he also suggested an incomplete ERPT estimated at about 0.4 , higher during exchange rate depreciations than appreciations. Then he affirmed that the ERPT degree declined in the 1990s due to macroeconomic reforms, being lower in countries with higher income and more floating exchange rate regimes. Maka (2013) investigated the asymmetrical ERPT to inflation in Ghana by using a structural VAR model on monthly data from 1990M1 to 2011M12. Maka (2013) found that the pass-through was asymmetric, with depreciations having a significant and positive impact on consumer prices compared to appreciations. In addition, he indicated a complete pass-through to non-food prices but incomplete for food prices. Jooste and Jhaveri (2014) examined the time-varying ERPT in South Africa, where they suggested a declining pass-through over time under a low inflationary environment. They also found that the ERPT was high during periods of exchange rate volatility. The ERPT to prices has been also studied in Malawi (Jombo et al. 2014) and Nigeria (Bada et al. 2016), revealing a lower pass-through in these countries. Thus, a striking feature of the studies on ERPT in the SSA region is that most of them are at the country level and neglect the possibility of nonlinearity and asymmetry in the relationship between exchange rate changes and domestic prices, as well as the cross-sectional dependence between countries. Hence, our study aims to fill this gap in the ERPT literature in the SSA region by addressing these issues.

\section{Data and Methodology}

\subsection{Data and Model Specification}

This study examines the exchange rate pass-through (ERPT) to the consumer price index (CPI) on quarterly data from 1990Q1 to 2017Q4 by using databases from International Financial Statistics (IFS) and World Development Indicators (WDI). This paper utilizes unbalanced panel data covering 40 sub-Saharan African (SSA) countries separated into 2 main groups. The first group is the CFA franc zone (14 countries), with a fixed exchange rate regime, comprising the Central African Economic and Monetary Community (CEMAC, 6 countries) and the West African Economic and Monetary Union (WAEMU, 8 countries). The second group encompasses the remaining countries, with a floating exchange rate regime (26 countries) and excluding the CEMAC and WAEMU subregions. Our sample is made up of heterogeneous data comprising different periods, with the longest ranging from 1990Q1 
to 2017Q4 and the shortest from 2007Q1 to 2017Q4, which gives a sample size ranging from 44 to 112 observations. This is mainly due to data availability for some countries. One way to deal with this problem is to use a wide range of panel units and appropriate econometric techniques to increase the reliability of the results.

As a starting point, we employed a modified specification of Delatte and Lòpez-Villavicencio (2012) and Brun-Aguerre et al. (2016). Especially, we formulated and adjusted our empirical model following the framework of Mohsen Bahmani-Oskooee and Amirhossein Mohammadian (Bahmani-Oskooee and Mohammadian 2017) in order to investigate the relationship between exchange rates and consumer prices in sub-Saharan Africa:

$$
l c p i_{i, t}=\alpha_{i}+\Theta_{i} l e r_{i, t}+\pi_{i} \operatorname{lmon}_{i, t}+\kappa_{i} \lg d p_{i, t}+\psi_{i} l o i l_{i, t}+\varepsilon_{i, t}
$$

where lcpi, ler, lmon, lgdp, and loil are the logarithmic transformations of the consumer price index (cpi), nominal exchange rate (er, the amount of local currency needed for 1 US dollar), money supply (mon), gross domestic product ( $g d p)$, and price of crude oil (oil, a proxy for foreign price), respectively; $\alpha_{i}, \Theta_{i}, \pi_{i}, \kappa_{i}$, and $\psi_{i}$ are the parameters for country $i$, and $\varepsilon$ is the error term. We used quarterly data from International Financial Statistics (IFS) for the consumer price index, nominal exchange rate, and crude oil price. We utilized annual data for gross domestic product (GDP) and money supply (broad money, as \% of GDP) from World Development Indicators (WDI), because data for these variables are not available on a quarterly basis for the whole sample. Then, we converted the annual data into the quarterly data by employing the low- to high-frequency technique. In fact, the conversion was done by using the quadratic-match average option of the low- to high-frequency method following Sbia Rashid and Hamdi (2014) and Shahbaz et al. (2017). This method performs an interpolation fitting a local quadratic polynomial such that the average of four adjacent quarters equals the data observed in the corresponding year. The subscripts $i$ and $t$ denote country and time, respectively.

We then followed the nonlinear autoregressive distributed lag (NARDL) approach developed by Shin et al. (2014) to investigate whether there is long-term co-integration and an asymmetrical relationship between the consumer price index (inflation) and exchange rate in the SSA countries. Some advantages of this approach are its suitability for a small sample size and its good performance in the presence of variables that are not integrated in the same order $(\mathrm{I}(0)$ or $\mathrm{I}(1))$. In addition, this framework has another advantage for testing short- and long-term nonlinearities in the relationship between variables through positive and negative partial sum decompositions of exogenous variables. However, the NARDL framework cannot be employed for second-order integrated variables.

Our NARDL model is a modified version of the autoregressive distributed lag (ARDL) following Campa and Goldberg (2005); Delatte and Lòpez-Villavicencio (2012); Brun-Aguerre et al. (2016); and Mohsen Bahmani-Oskooee and Amirhossein Mohammadian (Bahmani-Oskooee and Mohammadian 2017). We allow nonlinearity and asymmetry as follows:

$$
\begin{aligned}
\Delta l c p i_{i, t}= & \alpha_{i}+\beta_{i} l c p i_{i, t-1}+\Theta_{i}^{+} \operatorname{ler}_{i, t-1}^{+}+\Theta_{i}^{-} \operatorname{ler}_{i, t-1}^{-}+\zeta_{i} \operatorname{lmon}_{i, t-1}+\vartheta_{i} g a p_{i, t-1}+\eta_{i} \operatorname{loil}_{i, t-1}+\sum_{k=1}^{m-1} \vartheta_{i, k} \Delta l c p i_{i, t-k} \\
& +\sum_{k=0}^{n-1} \theta_{i, k}^{+} \Delta l e r_{i, t-k}^{+}+\sum_{k=0}^{p-1} \theta_{i, k}^{-} \Delta l e r_{i, t-k}^{-}+\sum_{k=0}^{q-1} \chi_{i, k} \Delta \operatorname{lmon}_{i, t-k}+\sum_{k=0}^{r-1} \mu_{i, k} \Delta g a p_{i, t-k}+\sum_{k=0}^{s-1} \sigma_{i, k} \Delta l o i i_{i, t-k}+\xi_{i, t}
\end{aligned}
$$

where $\Delta$ is the difference operator with an optimal lag order, gap is the output gap, ler ${ }^{+}$and ler ${ }^{-}$ represent positive and negative partial sums of exchange rate, denoting local currency depreciations (positive exchange rate changes) and appreciations (negative exchange rate changes), respectively; $\alpha_{i}$ refers to each country's specific intercept; $\beta_{i}, \Theta_{i}^{+}, \Theta_{i}^{-}, \zeta_{i}, \vartheta_{i}, \eta_{i}, \theta_{i}^{+}, \theta_{i}^{-}, \chi_{i}, \mu_{i}$ and $\sigma_{i}$ are coefficients to be estimated, and $\xi_{i, t} \sim \operatorname{IID}\left(0, \sigma^{2}\right) ; m, n, p, q, r$, and $s$ are the optimal lags based on the general-to-specific approach and the Akaike information criterion (AIC). We used a maximum lag 4 and removed the non-significant lagged variables by using this approach.

In addition, deviation of the nominal GDP from its Hodrick-Prescott filtered trend is utilized to compute the output gap variable (gap). With regard to Equation (2), the long-term ERPT elasticities 
are calculated by: $\Omega^{+}=-\left(\Theta_{i}^{+} / \beta_{i}\right)$ and $\Omega^{-}=-\left(\Theta_{i}^{-} / \beta_{i}\right)$, showing the long-term effects of depreciation and appreciation, respectively, of the local currency on domestic price. On the contrary, $\theta_{i, k}^{+}$and $\theta_{i, k}^{-}$are coefficients indicating the short-term effects of depreciation and appreciation on the consumer price index.

Following the modeling framework of Shin et al. (2014), the decompositions of the partial sum of exchange rate variable (ler) into positive changes $\left(l e r^{+}\right)$and negative changes $\left(l e r^{-}\right)$are computed as follows:

$$
\text { ler } r_{i, t}^{+}=\sum_{k=1}^{t} \Delta l e r_{i, k}^{+}=\sum_{k=1}^{t} \max \left(\Delta l e r_{i, k}, 0\right) \quad \text { and } \quad l e r_{i, t}^{-}=\sum_{k=1}^{t} \Delta l e r_{i, k}^{-}=\sum_{j=1}^{t} \min \left(\Delta l e r_{i, k}, 0\right),
$$

where $l e r_{i, t}^{+}$and $l e r_{i, t}^{-}$denote local currency depreciation and appreciation effects, respectively.

\subsection{Exchange Rate Pass-Through Estimation Per Country}

Our analysis was conducted following 3 steps. First, we performed 3 common unit root tests on each variable per country: augmented Dickey-Fuller (ADF, Dickey and Fuller 1981), Phillips-Perron (PP, Phillips and Perron 1988) and Kwiatkowski-Phillips-Schmidt-Shin (KPSS, Kwiatkowski et al. 1992). These tests were carried out after analyzing the descriptive statistics of the variables. The null hypothesis $\left(\mathrm{H}_{0}\right)$ of the ADF and PP tests supports the evidence of nonstationary variable against the alternative $\left(\mathrm{H}_{1}\right)$ of stationary variable, whereas KPSS tests the null hypothesis $\left(\mathrm{H}_{0}\right)$ of a stationary variable against the alternative $\left(\mathrm{H}_{1}\right)$ of nonstationary variable. Second, we estimated the NARDL model (Equation (2)) for each country following the general-to-specific method with a maximum lag length of 4, dropping all insignificant variables from the model.

We then investigated the existence of a long-term relationship in the model of Equation (2) using 2 approaches: Banerjee et al. (1998) $t$-test and Pesaran et al. (2001) F-test. The former $\left(t_{B D M}\right)$ tests the null hypothesis of $\beta_{i}=0$ against the one-sided alternative hypothesis $\beta_{i}<0$, while the latter $\left(F_{P S S}\right)$ tests the null hypothesis of no co-integration, i.e., $\beta_{i}=\Theta_{i}^{+}=\Theta_{i}^{-}=\zeta_{i}=\vartheta_{i}=\eta_{i}=0$, against the alternative of evidence of a long-term relationship: $\beta_{i} \neq \Theta_{i}^{+} \neq \Theta_{i}^{-} \neq \zeta_{i} \neq \vartheta_{i} \neq \eta_{i} \neq 0$.

We compared the computed t-test and F-test to the critical values of Banerjee et al. (1998) and Pesaran et al. (2001), respectively. As a guideline for the tests, there is a long-term relationship between the variables if the computed t-statistic and F-statistic are greater than the upper critical values found in Banerjee et al. (1998) and Pesaran et al. (2001). On the contrary, the evidence of no long-term co-integration cannot be rejected when the t-statistic and F-statistic fall below the respective lower critical bounds. Moreover, we tested the general hypothesis of symmetric exchange rate changes in model (2) in both the long and short term by performing Wald tests, $\Omega^{+}=\Omega^{-}$and $\sum_{k=0}^{n-1} \theta_{i, k}^{+}=\sum_{k=0}^{\theta-1} \theta_{i, k^{\prime}}^{-}$ respectively. Thus, Equation (2) can be rewritten with some restrictions allowing the evidence of either long-term symmetry, short-term symmetry, or both with respect to the results of Wald tests. When the results of Wald tests cannot reject the hypothesis of long-term symmetry, the NARDL model (Equation (2)) is rewritten as follows:

$$
\begin{aligned}
& \Delta l c p i_{i, t}=\alpha_{i}+\beta_{i} \text { lcpi }_{i, t-1}+\Theta_{i} \text { ler }_{i, t-1}+\zeta_{i} \text { lmon }_{i, t-1}+\vartheta_{i} \text { gap }_{i, t-1}+\eta_{i} \text { loil }_{i, t-1}+\sum_{k=1}^{m-1} \vartheta_{i, k} \Delta l c p i_{i, t-k} \\
& +\sum_{k=0}^{n-1} \theta_{i, k}^{+} \Delta l e r_{i, t-k}^{+}+\sum_{k=0}^{p-1} \theta_{i, k}^{-} \Delta l e r_{i, t-k}^{-}+\sum_{k=0}^{q-1} \chi_{i, k} \Delta \operatorname{lmon}_{i, t-k}+\sum_{k=0}^{r-1} \mu_{i, k} \Delta \operatorname{sap}_{i, t-k}+\sum_{k=0}^{s-1} \sigma_{i, k} \Delta \operatorname{loil}_{i, t-k}+\xi_{i, t}
\end{aligned}
$$

where Equation (3) describes the long-term symmetry between the exchange rate and the consumer price index with short-term asymmetry. The two other restricted NARDL models can be formulated by:

$$
\begin{aligned}
\Delta l c p i_{i, t}=\alpha_{i}+\beta_{i} l c p i_{i, t-1}+\Theta_{i}^{+} \operatorname{ler}_{i, t-1}^{+}+\Theta_{i}^{-} \operatorname{ler}_{i, t-1}^{-}+\zeta_{i} \operatorname{lmon}_{i, t-1}+\vartheta_{i} g a p_{i, t-1}+\eta_{i} l o i l_{i, t-1}+\sum_{k=1}^{m-1} \vartheta_{i, k} \Delta l c p i_{i, t-k} \\
+\sum_{k=0}^{n-1} \theta_{i, k} \Delta l e r_{i, t-k}+\sum_{k=0}^{q-1} \chi_{i, k} \Delta \operatorname{lmon}_{i, t-k}+\sum_{k=0}^{r-1} \mu_{i, k} \Delta g a p_{i, t-k}+\sum_{k=0}^{s-1} \sigma_{i, k} \Delta l o i l_{i, t-k}+\xi_{i, t}
\end{aligned}
$$




$$
\begin{aligned}
\Delta l c p i_{i, t}=\alpha_{i}+ & \Phi_{i} \text { lcpi }_{i, t-1}+\Theta_{i} \text { ler }_{i, t-1}+\zeta_{i} \text { lmon }_{i, t-1}+\vartheta_{i} \text { gap }_{i, t-1}+\eta_{i} \text { loil }_{i, t-1}+\sum_{k=1}^{m-1} \vartheta_{i, k} \Delta l c p i_{i, t-k} \\
& +\sum_{k=0}^{n-1} \theta_{i, k} \Delta l e r_{i, t-k}+\sum_{k=0}^{q-1} \chi_{i, k} \Delta \text { lmon }_{i, t-k}+\sum_{k=0}^{r-1} \mu_{i, k} \Delta g a p_{i, t-k}+\sum_{k=0}^{s-1} \sigma_{i, k} \Delta \text { loil }_{i, t-k}+\xi_{i, t}
\end{aligned}
$$

where Equation (4) shows the long-term asymmetry between exchange rate and local price change associated with short-term symmetry, while both long-term and short-term symmetries are depicted in Equation (5). Therefore, following Brun-Aguerre et al. (2016), we formulated 6 hypotheses:

Hypothesis 1. Supposes symmetric long-term ERPT, i.e., $H_{0}^{1}: \Omega_{i}^{+}=\Omega_{i}^{-}$against $H_{A}^{1}: \Omega_{i}^{+} \neq \Omega_{i}^{-}$.

Hypothesis 2. Assumes zero ERPT for depreciations $\left(\Omega_{i}^{+}\right)$or appreciations $\left(\Omega_{i}^{-}\right)$in the long term, i.e., $H_{0}^{2}$ : $\Omega_{i}^{+}=0, \quad\left(\Omega_{i}^{-}=0\right)$ against $H_{A}^{2}: \Omega_{i}^{+} \neq 0, \quad\left(\Omega_{i}^{-} \neq 0\right)$.

Hypothesis 3. Supposes complete long-term ERPT for depreciations or appreciations, $H_{0}^{3}$ : $\Omega_{i}^{+} \geq 1,\left(\Omega_{i}^{-} \geq 1\right)$ against $H_{A}^{3}: \Omega_{i}^{+}<1,\left(\Omega_{i}^{-}<1\right)$

Hypothesis 4. $H_{0}^{4}: \sum_{k=0}^{n-1} \theta_{i, k}^{+}=\sum_{k=0}^{\theta-1} \theta_{i, k}^{-} \quad$ against $H_{A}^{4}: \sum_{k=0}^{n-1} \theta_{i, k}^{+} \neq \sum_{k=0}^{\theta-1} \theta_{i, k}^{-}$.

Hypothesis 5. $H_{0}^{5}: \sum_{k=0}^{n-1} \theta_{i, k}^{+}=0, \quad\left(\sum_{k=0}^{\theta-1} \theta_{i, k}^{-}=0\right) \quad$ against $\quad H_{A}^{5}: \sum_{k=0}^{n-1} \theta_{i, k}^{+} \neq 0, \quad\left(\sum_{k=0}^{\theta-1} \theta_{i, k}^{-} \neq 0\right)$.

Hypothesis 6. $H_{0}^{6}: \sum_{k=0}^{n-1} \theta_{i, k}^{+} \geq 1, \quad\left(\sum_{k=0}^{\theta-1} \theta_{i, k}^{-} \geq 1\right) \quad$ against $\quad H_{A}^{6}: \sum_{k=0}^{n-1} \theta_{i, k}^{+}<1, \quad\left(\sum_{k=0}^{\theta-1} \theta_{i, k}^{-}<1\right)$.

Hypothesis 4 supports symmetric short-term ERPT, while Hypotheses 5 and 6 admit zero short-term and complete ERPT, respectively, with respect to depreciations and appreciations.

Finally, we estimated the appropriate NARDL models for each country based on the results of the symmetry test and recursively obtained the cumulative dynamic multipliers from the specific NARDL models employing the procedure of Shin et al. (2014) as follows:

$$
d m_{i, k}^{+}=\sum_{k=0}^{s} \frac{\partial d l c p i_{i, t+k}}{\partial d l e r_{i, t-1}^{+}} \quad, d m_{i, k}^{-}=\sum_{k=0}^{s} \frac{\partial d l c p i_{i, t+k}}{\partial d l e r_{i, t-1}^{-}}, \quad s=0,1,2, \ldots
$$

where $s \rightarrow \infty, d m_{i, k}^{+} \rightarrow \Omega^{+}$and $d m_{i, k}^{-} \rightarrow \Omega^{-}$with $\Omega^{+}$and $\Omega^{-}$are the long-term pass-through coefficients for depreciations and appreciations, respectively, defined above. The dynamic multipliers show the evolution of the consumer price index over time in response to a positive change (depreciation) and negative change (appreciation) of exchange rate.

\subsection{Panel Pass-Through Estimation}

In the next step, we first conducted a cross-sectional analysis of dependence between countries that can arise from unobserved common factors (psychological, economic, and social norms) using 4 well-known tests developed by Friedman (1937), Breusch and Adrian (1980), Frees (1995), and Pesaran (2004). Further details about these tests are provided in the Appendix A.

We implemented the panel ERPT using 2 common panel estimators: the feasible generalized least squares (FGLS) estimator of Parks (1967) and Kmenta (1986) and the pooled OLS regression with Driscoll and Kraay (1998) standard errors, which are convenient in the case of cross-sectional dependence (autocorrelation) and heteroscedasticity among the residuals between panel units. However, Parks and Kmenta's FGLS method is appropriate when the time period dimension (T) 
is greater than the cross-section dimension (K). In fact, the FGLS estimator becomes unworkable when $\mathrm{K}>\mathrm{T}$ because it will be impossible to get nonsingular estimated coefficients of the cross-sectional covariance matrix of type $\mathrm{K} \times \mathrm{K}$, or to produce acceptable standard error coefficients as argued by Beck and Katz (1995). Besides, Driscoll and Kraay (1998) approach is a nonparametric method to estimate standard errors that is robust to heteroscedasticity and autocorrelated errors between panel units, and can also be implemented for $\mathrm{T}<\mathrm{K}$, but not with a very small time period dimension, since this estimator is based on large $\mathrm{T}$ asymptotics. The approach of Driscoll and Kraay (1998) utilizes the Newey and West (1987) method to find the cross-sectional averages of the moment conditions. Finally, a pooled OLS regression using the standard errors of Driscoll and Kraay (1998) was performed following the procedure of Hoechle (2007), which is suitable for both balanced and unbalanced panel data and allows cross-sectional dependence across countries.

\section{Empirical Results and Discussion}

\subsection{Results of Pass-Through Estimations per Country}

The primary results start with some descriptive statistics on the variables for each country of our sample, as shown in Tables 1 and 2. The SSA region experiences more volatility in exchange rates across countries, as reflected by higher standard deviation values.

On the other hand, the same level of exchange rate volatility $(8.24 \%$, on average, in the CFA franc zone) in WAEMU and CEMAC countries can be explained by the fact that these regions belong to the CFA franc zone, with two currencies (West African CFA and XOF, and Central African CFA francs and $\mathrm{XAF}$ for WAEMU and CEMAC subregions, respectively).

Notably, the nominal exchange rate is more volatile in Congo Democratic Republic $(28.78 \%)$ and Angola (23.57\%) than in other countries, such as Cabo Verde (5.21\%) and Mauritius (4.24\%).

Although these two regions have a fixed exchange rate with respect to the euro and have always been at parity (so that they share a similar monetary value against other currencies), they are theoretically and institutionally separated and could have different monetary policies, which may influence the value of their respective currency at any time.

The results of Tables 1 and 2 show that most SSA countries present a common trend in exchange rate movements, since there have been more depreciations (positive changes in exchange rate) than appreciations (negative changes in exchange rate) of their local currency over the sample period. The magnitude of depreciation ranges from $45.45 \%$ (Comoros) to $97.33 \%$ (Ethiopia), except in the CFA franc zone (WAEMU and CEMAC), where the local currency depreciated by $51.98 \%$ on average across countries. This encourages more exporting from SSA countries due to the price competitiveness resulting from local currency depreciations. The results show a different pattern in price volatility, with higher price volatility in Congo Democratic Republic (19.85\%) and Angola (14.49\%) and relatively less pronounced effects in Namibia $(0.93 \%)$ and Botswana $(1.04 \%)$. Price deviation from mean value is mostly less than $5 \%$ in the remaining countries, especially in the CFA franc zone (around 3.54\% on average).

The results of the stationarity tests on each variable per country are depicted in Table 3. We performed traditional unit root tests as mentioned in Section 3 following a model with constant and no trend, and found evidence that all variables are almost stationary at first difference at any conventional significance level.

The nonlinear autoregressive distributed lag (NARDL) framework is justified to investigate the asymmetric exchange rate pass-through (ERPT) into prices, since no variable is second-order integrated. 
Table 1. Descriptive statistics for the CFA franc zone.

\begin{tabular}{|c|c|c|c|c|c|c|c|c|c|c|c|}
\hline \multirow[b]{3}{*}{ Country } & \multirow[b]{3}{*}{ Period } & \multicolumn{4}{|c|}{$\begin{array}{c}\text { Nominal Exchange Rate } \\
\text { (ler) }\end{array}$} & \multirow{2}{*}{\multicolumn{2}{|c|}{$\begin{array}{c}\begin{array}{c}\text { Consumer Price } \\
\text { Index } \\
(l c p i)\end{array} \\
d l c p i_{t}(\%)\end{array}$}} & \multirow{2}{*}{\multicolumn{2}{|c|}{$\begin{array}{c}\text { Output Gap } \\
\text { (gap) }\end{array}$}} & \multirow{2}{*}{\multicolumn{2}{|c|}{$\begin{array}{c}\begin{array}{c}\text { Money Supply } \\
\text { (lmon) }\end{array} \\
\text { dlmon }_{t}(\%)\end{array}$}} \\
\hline & & \multicolumn{4}{|c|}{$\operatorname{dler}_{t}(\%)$} & & & & & & \\
\hline & & Mean & Std. Dev & Depr (+) & Appr (-) & Mean & Std. Dev & Mean & Std. Dev & Mean & Std. Dev \\
\hline CFA franc zone (14) & 1990Q2-2017Q4 & 0.630 & 8.249 & 51.98 & 48.02 & 1.047 & 3.539 & -2.548 & 56.625 & 0.231 & 6.795 \\
\hline WAEMU $\left(K_{W}=8\right)$ & 1990Q2-2017Q4 & 0.595 & 8.197 & 51.48 & 48.52 & 1.067 & 3.664 & -3.451 & 40.905 & 0.185 & 7.601 \\
\hline Benin & 1992Q1-2017Q4 & 0.655 & 8.280 & 52.43 & 47.57 & 1.037 & 2.943 & -0.099 & 8.627 & 0.191 & 3.946 \\
\hline Burkina Faso & 1990Q1-2017Q4 & 0.587 & 8.224 & 51.35 & 48.65 & 0.719 & 2.241 & -0.118 & 8.419 & 0.839 & 4.099 \\
\hline Cote d'Ivoire & 1990Q1-2017Q4 & 0.587 & 8.224 & 51.35 & 48.65 & 0.886 & 2.027 & -0.012 & 2.840 & 0.201 & 4.067 \\
\hline Guinea-Bissau & 1990Q1-2017Q4 & 0.587 & 8.224 & 51.35 & 48.65 & 2.994 & 6.314 & -26.854 & 111.282 & -1.312 & 18.486 \\
\hline Mali & 1990Q1-2017Q4 & 0.587 & 8.224 & 51.35 & 48.65 & 0.691 & 2.770 & -0.052 & 4.843 & 0.164 & 4.038 \\
\hline Niger & 1990Q1-2017Q4 & 0.587 & 8.224 & 51.35 & 48.65 & 0.679 & 3.234 & -0.194 & 9.586 & 0.243 & 5.730 \\
\hline Senegal & 1990Q1-2017Q4 & 0.587 & 8.224 & 51.35 & 48.65 & 0.627 & 2.522 & -0.042 & 4.232 & 0.726 & 2.392 \\
\hline Togo & 1990Q1-2017Q4 & 0.587 & 8.224 & 51.35 & 48.65 & 0.896 & 4.646 & -0.000 & 8.556 & 0.434 & 3.768 \\
\hline CEMAC $\left(K_{C}=6\right)$ & 1990Q2-2017Q4 & 0.679 & 8.326 & 52.66 & 47.34 & 1.018 & 3.361 & -1.303 & 72.963 & 0.295 & 5.498 \\
\hline Cameroon & 1990Q1-2017Q4 & 0.587 & 8.224 & 51.35 & 48.65 & 0.808 & 2.173 & -0.025 & 2.820 & 0.000 & 3.463 \\
\hline Central African Rep. & 1990Q1-2015Q4 & 0.727 & 8.463 & 53.40 & 46.60 & 1.404 & 3.285 & -8.599 & 180.258 & 0.501 & 4.888 \\
\hline Chad & 1990Q1-2015Q4 & 0.727 & 8.463 & 53.40 & 46.60 & 0.961 & 4.902 & -0.369 & 13.719 & -0.000 & 4.807 \\
\hline Congo Rep. & 1990Q1-2015Q4 & 0.727 & 8.463 & 53.40 & 46.60 & 1.085 & 3.248 & -0.181 & 11.021 & 0.736 & 4.067 \\
\hline Equatorial Guinea & 1990Q1-2017Q4 & 0.587 & 8.224 & 51.35 & 48.65 & 1.332 & 3.214 & 1.085 & 17.733 & 0.104 & 9.688 \\
\hline Gabon & 1990Q1-2016Q4 & 0.730 & 8.334 & 53.27 & 46.73 & 0.532 & 2.837 & -0.058 & 4.248 & 0.456 & 3.192 \\
\hline Oil price $\left(\right.$ dloil $\left._{t}(\%)\right)$ & 1990Q1-2017Q4 & 0.793 & 8.326 & & & & & & & & \\
\hline
\end{tabular}

Note: $d$ is the first difference operator of the variable in the logarithmic form i.e., $d l e r_{t}=l e r_{t}-l e r_{t-1} ; d l c p i_{t}=l c p i_{t}-l c p i_{t-1} ; d l m o n_{t}=l m o n_{t}-l m o n_{t-1}$ and $d l o i l_{t}=l o l_{t}-l o i l_{t-1} ;$ Std. $_{\text {. Dev is }}$ the standard deviation of the variable; Depr (+) and Appr $(-)$ respectively represent the depreciation and the appreciation of the currency i.e., the percentage of the quarter in which there are positive (Depr $(+))$ and negative (Appr $(-)$ ) exchange rate changes. K is the number of the panel units. WAEMU denotes the West African Economic and Monetary Union, whereas CEMAC represents the Economic and Monetary Community of Central Africa. Authors' calculations using Eviews 9. 
Table 2. Descriptive statistics for the other Sub-Saharan African Countries.

\begin{tabular}{|c|c|c|c|c|c|c|c|c|c|c|c|}
\hline \multirow[b]{3}{*}{ Country } & \multirow[b]{3}{*}{ Period } & \multicolumn{4}{|c|}{$\begin{array}{c}\text { Nominal Exchange Rate } \\
(\text { ler })\end{array}$} & \multirow{2}{*}{\multicolumn{2}{|c|}{$\begin{array}{c}\text { Consumer Price Index } \\
\left(l_{c p i}\right)\end{array}$}} & \multirow{2}{*}{\multicolumn{2}{|c|}{$\begin{array}{c}\text { Output Gap } \\
\text { (gap) }\end{array}$}} & \multirow{2}{*}{\multicolumn{2}{|c|}{$\begin{array}{c}\text { Money Supply } \\
(\text { lmon })^{-}\end{array}$}} \\
\hline & & \multicolumn{4}{|c|}{$\operatorname{dler}_{t}(\%)$} & & & & & & \\
\hline & & Mean & Std.Dev & Depr (+) & Appr (-) & Mean & Std. Dev & Mean & Std. Dev & Mean & Std. Dev \\
\hline Other SSA $\left(K_{O}=26\right)$ & & 2.61 & 10.919 & 67.78 & 32.22 & 3.135 & 6.327 & -2.157 & 114.791 & 0.502 & 4.208 \\
\hline Angola & 1996Q1-2017Q4 & 9.575 & 23.576 & 87.36 & 12.64 & 11.872 & 14.491 & -0.062 & 3.777 & -0.15 & 5.775 \\
\hline Botswana & 1990Q1-2017Q4 & 1.484 & 4.996 & 66.67 & 33.33 & 1.99 & 1.045 & -0.022 & 3.243 & 0.691 & 5.07 \\
\hline Cabo Verde & 1990Q1-2017Q4 & 0.196 & 5.217 & 52.25 & 47.75 & 0.776 & 1.789 & -6.168 & 56.87 & 0.99 & 2.489 \\
\hline Comoros & 2000Q1-2013Q4 & -0.667 & 5.188 & 45.45 & 54.55 & 0.799 & 3.659 & 0.03 & 5.457 & 1.615 & 3.677 \\
\hline Congo, D. Rep & 1995Q1-2016Q4 & 11.864 & 28.785 & 77.01 & 22.99 & 11.203 & 19.856 & -0.14 & 5.218 & 0.837 & 5.129 \\
\hline Ethiopia & 1990Q1-2008Q4 & 2.094 & 10.249 & 97.33 & 2.67 & 2.127 & 4.69 & -0.054 & 3.771 & 0.131 & 3.381 \\
\hline Gambia & 1990Q1-2014Q4 & 1.708 & 6.41 & 69.7 & 30.3 & 1.373 & 1.684 & -2.022 & 22.038 & 0.748 & 5.737 \\
\hline Ghana & 1990Q1-2017Q4 & 4.497 & 6.545 & 96.4 & 3.6 & 4.341 & 3.588 & -0.05 & 3.903 & 0.701 & 3.737 \\
\hline Guinea & 2004Q1-2016Q4 & 2.997 & 8.574 & 79.39 & 19.61 & 3.822 & 2.933 & -0.027 & 5.971 & 0.506 & 5.907 \\
\hline Kenya & 1990Q1-2017Q4 & 1.353 & 6.414 & 59.46 & 40.54 & 2.758 & 3.334 & -0.036 & 3.076 & 0.26 & 2.424 \\
\hline Lesotho & 1990Q1-2017Q4 & 1.385 & 7.069 & 60.36 & 39.64 & 1.867 & 2.975 & -36.104 & 452.383 & -0.098 & 3.014 \\
\hline Liberia & 2001Q1-2015Q4 & 1.127 & 7.538 & 72.88 & 27.12 & 2.411 & 3.246 & 40.344 & 312.087 & 1.72 & 5.73 \\
\hline Madagascar & 1990Q1-2017Q4 & 2.117 & 8.882 & 57.66 & 42.34 & 2.726 & 3.415 & -0.03 & 5.431 & 0.646 & 3.136 \\
\hline Malawi & 1990Q1-2016Q4 & 5.158 & 11.082 & 76.64 & 23.36 & 4.733 & 7.091 & -0.179 & 12.304 & 0.157 & 6.208 \\
\hline Mauritius & 1990Q1-2017Q4 & 0.72 & 4.247 & 61.26 & 38.74 & 1.335 & 1.24 & -0.028 & 2.638 & 0.574 & 1.444 \\
\hline Mozambique & 2007Q1-2017Q4 & 1.885 & 7.272 & 60.47 & 39.53 & 1.99 & 2.408 & -0.043 & 4.029 & 1.286 & 2.901 \\
\hline Namibia & 2002Q1-2015Q4 & 0.133 & 7.704 & 49.21 & 50.79 & 1.432 & 0.931 & -61.524 & 3.719 & 0.6 & 3.986 \\
\hline Nigeria & 1990Q1-2016Q4 & 3.409 & 15.658 & 71.03 & 28.97 & 4.117 & 4.544 & -0.091 & 4.9 & 0.132 & 7.624 \\
\hline Rwanda & 1990Q1-2016Q4 & 2.187 & 7.755 & 67.29 & 32.71 & 1.891 & 2.594 & -1.113 & 20.016 & 0.254 & 4.436 \\
\hline Sao T. and Prin. & 2001Q1-2017Q4 & 1.264 & 4.147 & 64.18 & 35.82 & 2.989 & 2.061 & -0.002 & 3.482 & 0.379 & 3.395 \\
\hline Seychelles & 1990Q1-2017Q4 & 0.82 & 8.297 & 65.77 & 34.23 & 0.934 & 3.384 & 10.771 & 160.238 & 0.695 & 3.484 \\
\hline South Africa & 1990Q1-2017Q4 & 1.385 & 7.069 & 60.36 & 39.64 & 1.63 & 1.075 & -0.012 & 1.601 & 0.322 & 1.431 \\
\hline Swaziland & 1990Q1-2016Q4 & 1.534 & 7.108 & 61.68 & 38.32 & 1.912 & 2.057 & -0.577 & 11.681 & 0.401 & 3.158 \\
\hline Tanzania & 1990Q1-2016Q4 & 2.258 & 4.61 & 73.83 & 26.17 & 2.946 & 4.583 & -0.091 & 4.079 & 0 & 2.363 \\
\hline Uganda & 1993Q1-2017Q4 & 1.102 & 4.946 & 59.6 & 40.4 & 1.574 & 2.046 & -0.116 & 3.694 & 0.941 & 3.477 \\
\hline Zambia & 1990Q1-2017Q4 & 5.226 & 15.458 & 67.57 & 32.43 & 6.35 & 7.394 & -0.103 & 4.264 & 0.136 & 4.439 \\
\hline All SSA countries & 1990Q1-2017Q4 & 1.849 & 10.023 & 61.7 & 38.3 & 2.331 & 5.52 & -2.307 & 96.65 & 0.398 & 5.353 \\
\hline Oil price $\left(\right.$ dloil $\left._{t}(\%)\right)$ & 1990Q1-2017Q4 & 0.793 & 8.326 & & & & & & & & \\
\hline
\end{tabular}

Note: see Table 1. 
Table 3. Unit Root Tests.

\begin{tabular}{|c|c|c|c|c|c|c|c|c|c|c|c|c|}
\hline \multirow{4}{*}{ Country } & \multicolumn{3}{|c|}{$\begin{array}{c}\text { Nominal Exchange Rate } \\
\text { (ler) }\end{array}$} & \multicolumn{3}{|c|}{$\begin{array}{l}\text { Consumer Price Index } \\
\text { (lcpi) }\end{array}$} & \multicolumn{3}{|c|}{$\begin{array}{l}\text { Output Gap } \\
\underset{\text { (gap) }}{ }\end{array}$} & \multicolumn{3}{|c|}{$\begin{array}{l}\text { Money Supply } \\
\quad \text { (lmon) }\end{array}$} \\
\hline & \multicolumn{3}{|c|}{$\operatorname{ler}_{t}$} & \multicolumn{3}{|c|}{ lcpit } & \multicolumn{3}{|c|}{$\operatorname{gap}_{t}$} & \multicolumn{3}{|c|}{$\operatorname{lmon}_{t}$} \\
\hline & ADF: & PP & KPSS & ADF: & PP & KPSS & ADF: & PP & KPSS & ADF: & PP & KPSS \\
\hline & $H_{0}=I(\mathbf{1})$ & $\mathrm{H}_{0}=\mathrm{I}(\mathbf{1})$ & $H_{0}=I(0)$ & $\mathrm{H}_{0}=\mathrm{I}(\mathbf{1})$ & $H_{0}=I(1)$ & $\mathrm{H}_{0}=\mathrm{I}(\mathbf{0})$ & $\mathrm{H}_{0}=\mathrm{I}(\mathbf{1})$ & $\mathrm{H}_{0}=\mathrm{I}(\mathbf{1})$ & $\mathrm{H}_{0}=\mathrm{I}(\mathbf{0})$ & $\mathrm{H}_{0}=\mathrm{I}(\mathbf{1})$ & $\mathrm{H}_{0}=\mathrm{I}(\mathbf{1})$ & $\mathrm{H}_{0}=\mathrm{I}(\mathbf{0})$ \\
\hline Benin & $-2.916^{* *}$ & $-2.874^{* * *}$ & 0.201 & $-3.352^{* *}$ & $-3.242 * *$ & $1.152 *$ & $-5.434^{*}$ & $-3.225^{* *}$ & 0.025 & -1.173 & -0.79 & $0.843 *$ \\
\hline Burkina Faso & -2.235 & -2.21 & $0.357^{* * *}$ & -1.935 & -1.693 & 1.140 * & $-4.687^{*}$ & $-3.841 *$ & 0.025 & 0.87 & 0.22 & 0.923 * \\
\hline Cote d'Ivoire & -2.235 & -2.21 & $0.357^{* * *}$ & $-3.045^{* *}$ & -2.344 & 1.127 * & -4.369 * & $-3.575 *$ & 0.026 & -1.06 & -0.812 & $0.559 * *$ \\
\hline Guinea-Bissau & -2.235 & -2.21 & $0.357^{* * *}$ & $-3.383^{* *}$ & $7.297^{*}$ & 0.911 * & $-4.937^{*}$ & $-4.412 *$ & 0.263 & -1.623 & $-3.692 *$ & $0.512 * *$ \\
\hline Mali & -2.235 & -2.21 & $0.357^{* * *}$ & $-2.785^{* * *}$ & -1.465 & $1.122 *$ & $-3.060 * *$ & $-2.746^{* * *}$ & 0.029 & -2.395 & -2.4 & 0.749 \\
\hline Niger & -2.235 & -2.21 & $0.357^{* * *}$ & $-2.585^{* * *}$ & -1.221 & 1.081 * & $-4.739 *$ & $-3.786 *$ & 0.027 & -1.104 & -0.665 & 0.520 ** \\
\hline Senegal & -2.235 & -2.21 & $0.357^{* * *}$ & -1.763 & -1.697 & 1.081 * & $-4.345^{*}$ & $-3.846^{*}$ & 0.027 & 1.72 & 0.624 & $1.093 *$ \\
\hline Togo & -2.235 & -2.21 & $0.357^{* * *}$ & -2.023 & -1.655 & $1.063 *$ & $-4.924 *$ & $-3.434 *$ & 0.025 & 0.074 & -0.125 & $0.772 *$ \\
\hline Cameroon & -2.235 & -2.21 & $0.357^{* * *}$ & -1.93 & -1.661 & 1.121 & $-5.832 *$ & -3.877 * & 0.028 & -1.644 & -1.237 & 0.518 ** \\
\hline Central African Rep. & -2.076 & -2.048 & $0.374^{* * *}$ & 1.496 & 1.532 & 1.17 & $-9.055 *$ & $-9.014 *$ & 0.045 & -1.938 & -1.3 & 0.234 \\
\hline Chad & -2.076 & -2.048 & $0.374^{* * *}$ & -1.904 & -1.452 & 1.141 & $-4.718 *$ & $-2.950 * *$ & 0.038 & -1.252 & -2.236 & 0.191 \\
\hline Congo Rep. & -2.076 & -2.048 & $0.374^{* * *}$ & -1.165 & -1.124 & 1.108 & $-3.486^{* *}$ & $-3.435^{* *}$ & 0.022 & 0.252 & 2.611 & 0.62 \\
\hline Equatorial Guinea & -2.076 & -2.048 & $0.374^{* * *}$ & $-5.251 *$ & -1.577 & 1.177 & $-7.638 *$ & $-7.638 *$ & 0.041 & -0.299 & -1.648 & 0.344 \\
\hline Gabon & -2.094 & -2.062 & $0.382 * * *$ & -0.842 & -0.898 & 1.014 & $-2.697^{* * *}$ & $-3.259 * *$ & 0.03 & -0.569 & -0.983 & 0.828 \\
\hline Angola & $-6.989 *$ & -5.277 * & $0.843 *$ & -2.464 & $-7.545^{*}$ & $1.000 *$ & $-3.496^{* *}$ & $-3.398^{* *}$ & 0.049 & -1.919 & -1.333 & $0.766 *$ \\
\hline Botswana & -1.488 & -1.468 & 1.140 * & -1.241 & $-4.866^{*}$ & $1.220 *$ & -2.445 & $-3.375^{* *}$ & 0.029 & -1.02 & -1.895 & 0.891 * \\
\hline Cabo Verde & -1.935 & -1.951 & 0.193 & -1.853 & $-3.923 *$ & $1.156^{*}$ & $-5.907 *$ & $-9.570 *$ & 0.445 & -2.089 & -2.077 & $1.008 *$ \\
\hline Comoros & -1.547 & -1.547 & $0.649^{* *}$ & -1.779 & -1.453 & $0.896^{*}$ & $-4.281 *$ & $-2.779 * * *$ & 0.05 & -0.82 & $-2.837 * * *$ & 0.889 \\
\hline Congo Dem. Rep. & -2.121 & $-4.526^{*}$ & $0.948 *$ & $-4.518 *$ & $-4.265^{*}$ & 0.943 * & $-2.695^{* * *}$ & $-3.438^{* *}$ & 0.056 & -0.824 & 0.078 & 0.852 \\
\hline Ethiopia & -1.124 & -2.178 & $0.909 *$ & 0.138 & 0.565 & 0.996 * & $-3.856^{*}$ & $-3.134^{* *}$ & 0.042 & -1.555 & -1.497 & $0.887 *$ \\
\hline Gambia & -0.462 & -0.109 & $1.130 *$ & -0.075 & -0.358 & $1.209 *$ & -2.48 & $-3.341^{* *}$ & 0.076 & -2.045 & -0.283 & $1.120 *$ \\
\hline Ghana & -2.052 & -1.922 & 1.163 * & $-2.918^{* *}$ & $-2.626^{* * *}$ & $1.192 *$ & $-3.845 *$ & $-3.461^{* *}$ & 0.033 & -1.973 & -2.504 & 0.917 * \\
\hline Guinea & -0.369 & $-2.656^{* * *}$ & 0.902 * & $-4.525 *$ & $-4.274 *$ & 0.953 * & $-3.899 *$ & -2.59 & 0.047 & -1.96 & -1.437 & 0.568 \\
\hline Kenya & $-2.974^{* *}$ & $-3.070^{* *}$ & 1.029 * & -1.999 & $-2.779 * *$ & 1.205 * & $-4.165 *$ & $-3.204^{* *}$ & 0.036 & $-2.614^{* * *}$ & $-2.742^{* * *}$ & $0.628^{* *}$ \\
\hline Lesotho & -1.697 & -1.329 & 1.025 * & -1.862 & $-2.690 * *$ & $1.052 *$ & $-11.187^{*}$ & -12.343 * & 0.129 & -1.435 & -2.547 & 0.243 * \\
\hline Liberia & -0.899 & -2.024 & $0.908 *$ & -0.911 & $-2.596^{* * *}$ & $0.970 *$ & $-7.664 *$ & $-7.665 *$ & 0.136 & $-3.930 *$ & -1.02 & $0.805 *$ \\
\hline Madagascar & -1.767 & -1.776 & $1.106 *$ & -2.088 & -2.137 & $1.184 *$ & $-3.741 *$ & -4.648 * & 0.023 & -1.006 & $-3.317^{* *}$ & 1.001 * \\
\hline Malawi & -2.185 & -1.053 & 1.119 * & -2.424 & -1.56 & $1.136^{*}$ & $-5.075 *$ & $-3.872 *$ & 0.025 & -1.737 & -1.049 & 0.299 \\
\hline Mauritius & -1.696 & -1.696 & 1.059 * & $-3.320 * *$ & $-3.222 * *$ & $1.217^{*}$ & -2.31 & $-3.670 *$ & 0.029 & -1.146 & -1.486 & 1.165 * \\
\hline Mozambique & -0.859 & -0.368 & $0.626^{* *}$ & -0.323 & -0.343 & 0.823 * & $-3.682 *$ & -2.561 & 0.076 & -1.172 & -1.916 & 0.763 * \\
\hline Namibia & -0.95 & -1.161 & $0.654^{* *}$ & -0.088 & -0.841 & 1.023 & $-5.108 *$ & $-4.446^{*}$ & $0.615^{\text {** }}$ & -1.711 & -1.393 & $0.684^{* *}$ \\
\hline Nigeria & -1.606 & -1.606 & $1.053 *$ & -1.984 & $-3.039 * *$ & $1.105 *$ & -3.624 * & $-3.084^{* *}$ & 0.038 & $-3.168^{* *}$ & $-2.617^{* * *}$ & 0.11 \\
\hline Rwanda & $-2.827^{* * *}$ & $-3.379^{* *}$ & 1.054 * & -0.849 & -1.72 & $1.200 *$ & $-3.588 *$ & $-3.059 * *$ & 0.041 & $-3.109 * *$ & -1.995 & $0.575^{* *}$ \\
\hline Sao Tome \& Prin. & -1.286 & -1.292 & 1.024 * & -1.843 & -1.827 & 1.051 * & $-4.318^{*}$ & $-3.111^{* *}$ & 0.042 & -2.565 & -2.397 & $0.611^{* *}$ \\
\hline
\end{tabular}


Table 3. Cont

\begin{tabular}{|c|c|c|c|c|c|c|c|c|c|c|c|c|}
\hline \multirow{4}{*}{ Country } & \multicolumn{3}{|c|}{$\begin{array}{c}\text { Nominal Exchange Rate } \\
\text { (ler) }\end{array}$} & \multicolumn{3}{|c|}{$\begin{array}{c}\text { Consumer Price Index } \\
\text { (lcpi) }\end{array}$} & \multicolumn{3}{|c|}{$\begin{array}{l}\text { Output Gap } \\
\text { (gap) }\end{array}$} & \multicolumn{3}{|c|}{$\begin{array}{c}\text { Money Supply } \\
\text { (lmon) }\end{array}$} \\
\hline & \multicolumn{3}{|c|}{$\operatorname{ler}_{t}$} & \multicolumn{3}{|c|}{ lcpi $i_{t}$} & \multicolumn{3}{|c|}{$\operatorname{gap}_{t}$} & \multicolumn{3}{|c|}{$\operatorname{lmon}_{t}$} \\
\hline & ADF: & PP & KPSS & ADF: & PP & KPSS & ADF: & PP & KPSS & ADF: & PP & KPSS \\
\hline & $H_{0}=I(\mathbf{1})$ & $\mathrm{H}_{0}=\mathrm{I}(\mathbf{1})$ & $\mathrm{H}_{0}=\mathrm{I}(\mathbf{0})$ & $\mathrm{H}_{0}=\mathrm{I}(\mathbf{1})$ & $\mathrm{H}_{0}=\mathrm{I}(\mathbf{1})$ & $\mathrm{H}_{0}=\mathrm{I}(\mathbf{0})$ & $\mathrm{H}_{0}=\mathrm{I}(\mathbf{1})$ & $\mathrm{H}_{0}=\mathrm{I}(\mathbf{1})$ & $\mathrm{H}_{0}=\mathrm{I}(0)$ & $\mathrm{H}_{0}=\mathrm{I}(\mathbf{1})$ & $\mathrm{H}_{0}=\mathrm{I}(\mathbf{1})$ & $\mathrm{H}_{0}=\mathrm{I}(\mathbf{0})$ \\
\hline Seychelles & -0.25 & -0.374 & $1.018^{*}$ & 0.047 & 0.219 & 1.108 * & $-5.102 *$ & $-5.132 *$ & 0.059 & -2.451 & -2.212 & 0.248 \\
\hline South Africa & -1.697 & -1.329 & $1.025 *$ & $-2.679 * * *$ & -3.577 * & 1.214 * & -3.591 * & $-3.008^{* *}$ & 0.036 & -1.394 & -0.957 & 1.040 * \\
\hline Swaziland & -0.99 & -1.019 & 0.991 * & -2.334 & -2.434 & 1.180 * & -3.564 * & $-3.298 * *$ & 0.043 & -0.706 & -0.567 & $0.371^{* * *}$ \\
\hline Tanzania & $-3.460 * *$ & $-3.020^{* *}$ & $1.122 *$ & $-2.626^{* * *}$ & -4.070 * & 1.104 * & -3.699 * & -3.945 * & 0.044 & $-3.365^{* *}$ & -2.054 & 0.308 \\
\hline Uganda & -0.512 & 0.116 & 1.095 * & 0.081 & 0.252 & 1.195 * & -2.507 & $-7.135^{*}$ & 0.058 & -2.071 & -0.125 & 0.772 * \\
\hline Zambia & $-5.974 *$ & $5.648^{*}$ & 0.976 * & $-5.107^{*}$ & $-8.642 *$ & 1.102 * & -3.561 * & $-3.236^{* *}$ & 0.045 & -2.071 & $-2.761^{* * *}$ & 1.125 * \\
\hline $\begin{array}{l}\text { Oil price } \\
\text { loil }_{t}\end{array}$ & $\begin{array}{l}\text { ADF: } \\
-1.174\end{array}$ & $\begin{array}{c}\mathrm{PP} \\
-1.011\end{array}$ & $\begin{array}{l}\text { KPSS } \\
0.973 *\end{array}$ & & & & & & & & & \\
\hline
\end{tabular}

Note: ler, lcpit, lmon and loil $t$ denote the logarithmic form of the nominal exchange rate, the consumer price index, the money supply and the crude oil price, respectively. ADF and PP respectively represent the Augmented Dickey-Fuller and Phillips-Perron unit root tests, while KPSS refers to the Kwiatkowski-Phillips-Schmidt-Shin test statistics. The null hypothesis $\mathrm{H}_{0}=\mathrm{I}(1)$ of the ADF and PP tests shows that the variable is stationary at the first difference with respect to the alternative hypothesis of stationarity at level. However, the null hypothesis $\mathrm{H}_{0}=\mathrm{I}(0)$ for the KPSS test implies that the variable is stationary at level against the alternative hypothesis of stationary variable at first difference. The symbols *,**,*** show the rejection of the null hypothesis respectively at $1 \%, 5 \%$ and $10 \%$ level of significance. Authors' calculations using Eviews 9 . 
Tables 4 and 5 show the outcomes of the co-integration analysis. The results are mixed, following the diverse specifications as well as the $t_{B D M}$ of Banerjee et al. (1998) and the $F_{P S S}$ of Pesaran et al. (2001) test statistics.

Table 4. Cointegration Tests for CFA Franc Zone.

\begin{tabular}{|c|c|c|c|c|c|c|c|c|}
\hline \multirow{3}{*}{ Countries } & \multicolumn{2}{|c|}{$\begin{array}{c}\text { Unrestricted NARDL } \\
\text { NARDL Model (2] }\end{array}$} & \multicolumn{6}{|c|}{ Restricted NARDLs } \\
\hline & \multicolumn{2}{|c|}{$\mathbf{S}_{\mathrm{T}} \mathbf{A} \& \mathbf{L}_{\mathrm{T}} \mathbf{A}$} & \multicolumn{2}{|c|}{$\mathrm{S}_{\mathrm{T}} \mathrm{A} \& \mathrm{~L}_{\mathrm{T}} \mathrm{S}$} & \multicolumn{2}{|c|}{$S_{T} S \& L_{T} A$} & \multicolumn{2}{|c|}{$\mathbf{S}_{\mathrm{T}} \mathrm{S} \& \mathrm{~L}_{\mathrm{T}} \mathrm{S}$} \\
\hline & $t_{B D M}$ & $F_{P S S}$ & $t_{B D M}$ & $F_{P S S}$ & $t_{B D M}$ & $F_{P S S}$ & $t_{B D M}$ & $F_{P S S}$ \\
\hline & \multicolumn{2}{|c|}{ Unrestricted NARDL } & \multicolumn{6}{|c|}{ Restricted NARDLs } \\
\hline Burkina Faso & $-4.314^{* *}$ & 3.346 & $-3.856^{* * *}$ & 3.213 & $-4.573^{* *}$ & $3.754^{* * *}$ & $-3.743 * * *$ & 3.092 \\
\hline Cote d'Ivoire & -1.917 & 2.123 & 0.285 & 1.880 & $-5.515^{*}$ & $5.934 *$ & -1.308 & 2.254 \\
\hline Guinea-Bissau & $-5.637^{*}$ & $8.895^{*}$ & $-6.024^{*}$ & $10.935 *$ & $-5.333^{*}$ & $8.164 *$ & $-5.718^{*}$ & $9.898 *$ \\
\hline Mali & $-7.187^{*}$ & 10.217 * & -3.215 & 2.900 & $-5.593 *$ & $7.371 *$ & -2.148 & 3.479 \\
\hline Niger & $-5.422 *$ & $5.552 *$ & $-5.167^{*}$ & $5.805 *$ & $-5.786 *$ & $6.128 *$ & $-5.302 *$ & $6.018 *$ \\
\hline \multicolumn{9}{|l|}{ CEMAC $\left(K_{C}=6\right)$} \\
\hline Central African Rep. & -0.532 & 3.399 & 0.838 & $4.377^{* *}$ & -1.133 & 1.592 & 0.328 & 2.444 \\
\hline Chad & $-6.597 *$ & $8.501 *$ & $-5.337^{*}$ & $7.000 *$ & $-6.057^{*}$ & $8.832 *$ & $-5.080 *$ & $6.460 *$ \\
\hline Congo Rep. & $-4.355^{* *}$ & $6.594 *$ & -2.719 & $4.540 * *$ & -2.787 & 2.443 & -1.650 & 2.748 \\
\hline Equatorial Guinea & $-5.902 *$ & $7.451 *$ & -2.203 & 3.412 & $-4.915^{*}$ & $5.845^{*}$ & -1.384 & 1.576 \\
\hline Gabon & $-4.602^{* *}$ & $6.210 *$ & -2.029 & 1.318 & -3.544 & 3.004 & -2.866 & 2.178 \\
\hline
\end{tabular}

Note: NARDL: nonlinear autoregressive distributed lag; $\mathrm{S}_{\mathrm{T}} \mathrm{A}$ : Short-Term Asymmetry; $\mathrm{L}_{\mathrm{T}} \mathrm{A}$ : Long-Term Asymmetry; $\mathrm{S}_{\mathrm{T}} \mathrm{S}$ : Short-Term Symmetry and $\mathrm{L}_{\mathrm{T}} \mathrm{S}$ denote Long-Term Symmetry models; $t_{B D M}$ and $F_{P S S}$ denote the Banerjee et al. (1998) $t$-test and the Pesaran et al. (2001) F-test respectively. Given the small sample size, we use the critical values of Narayan (2005) following the sample size of each country (see Appendix B, Table A1). For instance, we use the critical values with $n=80$ observations for countries with $n \geq 80$. For these countries, the critical values [lower bound: $\mathrm{I}(0)$; upper bound: $\mathrm{I}(1)]$ for $F_{P S S}$ test with $\mathrm{k}=5$ (k being the number of explanatory variables in the model namely $l e r^{-}, l e r^{+}$, lmon, gap and loil) for Equations (2) and (4) are [3.725; 5.163] at $1 \%,[2.787 ; 4.015]$ at $5 \%$ and $[2.355 ; 3.500]$ at $10 \%$ significance levels but the similar critical values with $\mathrm{k}=4$ (ler, lmon, gap and loil) for models (3) and (5) are [4.096; 5.512] at $1 \%,[3.010 . ; 4.216]$ at $5 \%$ and $[2.548 ; 3.644]$ at $10 \%$. The corresponding critical values for $t_{B D M}$ using t-bounds test with $\mathrm{k}=5$ for models $(2)$ and $(4)$ are: $[-3.43 ;-4.79]$ at $1 \%,[-2.86 ;-4.19]$ at $5 \%$ and $[-2.57 ;-3.86]$ at $10 \%$. The similar critical values for $t_{B D M}$ with $\mathrm{k}=4$ for Equations $(3)$ and $(5)$ are $[-3.43 ;-4.60]$ at $1 \%,[-2.86 ;-3.99]$ at $5 \%$ and $[-2.57 ;-3.66]$ at $10 \%$. Values reported in the table are t-statistics for $t_{B D M}$ test and F-statistics for $F_{P S S}$. The models have been estimated following the general-to-specific approach (uni-directional method and $p$-value backwards 10\% significance level as stopping criteria) with maximum lag length 4 (Campa and Goldberg 2005; Delatte and Lòpez-Villavicencio 2012). The symbols ${ }^{* * *}, * *$ and * show the significance at $10 \%, 5 \%$ and $1 \%$ levels, respectively. Authors' computations by using Eviews 9.

For instance, the evidence of long-term relationships among the variables depends on the model specification in many cases (Cote d'Ivoire, Mali, Central African Republic, Gabon, Liberia, South Africa, Uganda, etc.) and the significance of one or both $t_{B D M}$ and $F_{P S S}$ statistics (Botswana, Cabo Verde, Ethiopia, Madagascar, among others) compared with their critical bounds values. 
Table 5. Cointegration Tests for the other SSA Countries.

\begin{tabular}{|c|c|c|c|c|c|c|c|c|}
\hline & \multicolumn{2}{|c|}{$\begin{array}{l}\text { Unrestricted NARDL } \\
\text { NARDL Model (2) }\end{array}$} & \multicolumn{6}{|c|}{ Restricted NARDLs } \\
\hline & \multicolumn{2}{|c|}{$\mathrm{S}_{\mathrm{T}} \mathbf{A} \& \mathrm{~L}_{\mathrm{T}} \mathbf{A}$} & \multicolumn{2}{|c|}{$\begin{array}{c}\text { NARDL Model (3) } \\
S_{\mathrm{T}} \mathrm{A} \& \mathrm{~L}_{\mathrm{T}} \mathrm{S}\end{array}$} & \multicolumn{2}{|c|}{$\frac{\text { NARDL Model (4) }}{S_{\mathrm{T}} S \& \mathrm{~L}_{\mathrm{T}} \mathrm{A}}$} & \multicolumn{2}{|c|}{$\begin{array}{c}\text { NARDL Model (5) } \\
\mathrm{S}_{\mathrm{T}} \mathrm{S} \& \mathrm{~L}_{\mathrm{T}} \mathrm{S}\end{array}$} \\
\hline & $t_{B D M}$ & $F_{P S S}$ & $t_{B D M}$ & $F_{P S S}$ & $t_{B D M}$ & $F_{P S S}$ & $t_{B D M}$ & $F_{P S S}$ \\
\hline Other $\left(\mathrm{K}_{\mathrm{O}}=26\right)$ & & & & & & & & \\
\hline Angola & -0.797 & $14.627 *$ & -0.769 & $17.781 *$ & -2.674 & $9.344 *$ & -1.622 & 11.470 * \\
\hline Comoros & $-7.058^{*}$ & $10.040 *$ & $-5.265^{*}$ & $6.001 *$ & $-8.238 *$ & $11.705^{*}$ & $-6.497^{*}$ & $8.901 *$ \\
\hline Congo, Dem. Rep. & -3.246 & 16.544 * & -1.823 & $24.557 *$ & -0.226 & $4.757^{*}$ & 1.681 & 3.307 \\
\hline Ethiopia & -1.454 & $4.752^{* *}$ & 2.776 & $9.477 *$ & -1.588 & $4.257^{* *}$ & -1.806 & $3.781^{* * *}$ \\
\hline Gambia & $-5.105^{*}$ & $8.456^{*}$ & -3.547 & $6.682 *$ & $-5.617 *$ & $8.874^{*}$ & -2.865 & $5.082 *$ \\
\hline Ghana & -2.051 & 1.850 & -2.072 & 2.196 & -1.618 & 1.485 & -1.633 & 1.800 \\
\hline Madagascar & -0.524 & $5.870 *$ & -1.910 & $7.069 *$ & -3.301 & $5.745^{*}$ & -3.002 & $7.793 *$ \\
\hline Malawi & -3.672 & $7.609 *$ & $-3.686^{* * *}$ & $9.085 *$ & -2.363 & $4.740^{* *}$ & -2.293 & $5.571 *$ \\
\hline Mauritius & -2.683 & 2.801 & -2.223 & 2.325 & -2.751 & 2.792 & -1.802 & 2.438 \\
\hline Mozambique & -2.688 & $8.900 *$ & $-4.503^{* * *}$ & $11.843 *$ & -1.845 & $11.138 *$ & 0.565 & $6.294^{*}$ \\
\hline Namibia & -3.144 & $3.703^{* * *}$ & -0.978 & 1.449 & -1.735 & $4.289 * *$ & -0.401 & 1.392 \\
\hline Nigeria & $-6.595^{*}$ & $8.916^{*}$ & -2.039 & $6.912 *$ & -2.075 & $5.492 * * *$ & -2.154 & $6.189 *$ \\
\hline Rwanda & -2.981 & $3.501 * * *$ & $-4.612^{* *}$ & $5.470 * *$ & $-4.412^{* *}$ & $4.509 * *$ & $-4.612^{* *}$ & $5.470^{* *}$ \\
\hline Sao Tome and Prin. & -3.151 & $3.547^{* * *}$ & $-3.681^{* * *}$ & $4.355^{* *}$ & $-4.356^{* *}$ & $6.041 *$ & $-5.478^{*}$ & $7.374^{*}$ \\
\hline Seychelles & -1.719 & $6.984^{*}$ & -3.240 & $7.859 *$ & $-7.373 *$ & $15.031 *$ & $-7.098^{*}$ & $12.491 *$ \\
\hline South Africa & -3.345 & $3.561 * * *$ & -1.470 & 2.800 & -3.419 & $5.073^{* *}$ & -1.326 & 3.296 \\
\hline Swaziland & -1.922 & 2.293 & -1.759 & 2.478 & -1.922 & 2.293 & -1.759 & 2.478 \\
\hline
\end{tabular}

However, in at least one of the various NARDL models, the long-term relationship between consumer prices, exchange rates, money supply, output gap, and oil price cannot be rejected, since one or both $t_{B D M}$ and $F_{P S S}$ statistics are greater than the corresponding critical bounds values at the conventional level of significance in most cases. The results of the symmetry tests for each country in the short and long term are presented in Tables 6 and 7.

Table 6. Symmetry tests for CFA franc zone.

\begin{tabular}{|c|c|c|c|}
\hline Country & $\begin{array}{c}\text { Short-Term Tests }\left(\mathrm{W}_{\mathrm{ST}}\right) \text { : } \\
H_{0}: \sum_{k}^{s} \theta_{i, k}^{-}=\sum_{k}^{t} \theta_{i, k}^{+}\end{array}$ & $\begin{array}{c}\text { Long-Term Tests }\left(\mathrm{W}_{\mathrm{LT}}\right) \text { : } \\
H_{0}: \Omega^{-}=\Omega^{+}\end{array}$ & $\begin{array}{l}\text { Unrestricted \& Restricted } \\
\text { NARDL Models }\end{array}$ \\
\hline \multicolumn{4}{|l|}{ WAEMU $\left(\mathrm{K}_{\mathrm{W}}=8\right)$} \\
\hline $\operatorname{Benin}^{(a)}$ & $66.787 *$ & $6.121^{* *}$ & Rest. NARDL model (2) \\
\hline Burkina Faso $^{\text {(a) }}$ & 12.752 * & $4.921 * *$ & Unr. NARDL model (2) \\
\hline Cote d'Ivoire ${ }^{(c)}$ & - & $81.428 *$ & Rest. NARDL model (4) \\
\hline Guinea-Bissau (a) & 11.648 * & 0.001 & Rest. NARDL model (3) \\
\hline Mali $^{(a)}$ & $4.105^{* *}$ & $78.592 *$ & Rest. NARDL model (2) \\
\hline Niger ${ }^{(a)}$ & 1.348 & $0.213 *$ & Rest. NARDL model (4) \\
\hline Senegal (a) & -0.034 & 0.248 * & Unr. NARDL model (4) \\
\hline $\operatorname{Tog}_{0}{ }^{(a)}$ & 1.613 & $7.754 *$ & Unr NARDL model (4) \\
\hline
\end{tabular}


Table 6. Cont.

\begin{tabular}{|c|c|c|c|}
\hline Country & $\begin{array}{l}\text { Short-Term Tests }\left(\mathrm{W}_{\mathrm{ST}}\right) \text { : } \\
\qquad H_{0}: \sum_{k}^{s} \theta_{i, k}^{-}=\sum_{k}^{t} \theta_{i, k}^{+}\end{array}$ & $\begin{array}{c}\text { Long-Term Tests }\left(\mathrm{W}_{\mathrm{LT}}\right) \text { : } \\
H_{0}: \Omega^{-}=\Omega^{+}\end{array}$ & $\begin{array}{l}\text { Unrestricted \& Restricted } \\
\text { NARDL Models }\end{array}$ \\
\hline \multicolumn{4}{|l|}{ CEMAC $\left(K_{C}=6\right)$} \\
\hline Cameroon (a) & $66.843 *$ & $74.393 *$ & Unr. NARDL model (2) \\
\hline Central Afr. Rep ${ }^{(b)}$ & $24.061 *$ & - & Rest. NARDL model (3) \\
\hline Chad $^{(a)}$ & $20.088 *$ & $18.444 *$ & Rest. NARDL model (2) \\
\hline Congo Rep ${ }^{(a)}$ & $6.035 * *$ & $28.834 *$ & Rest. NARDL model (2) \\
\hline Equatorial Guinea (a) & $4.864^{* *}$ & $134.545^{*}$ & Unr. NARDL model (2) \\
\hline Gabon (a) & 22.314 * & $27.315 *$ & Rest. NARDL model (2) \\
\hline
\end{tabular}

Note: $\sum_{k}^{s} \theta_{i, k}^{-}\left(\sum_{k}^{t} \theta_{i, k}^{+}\right)$is the short-term additive pass-through for appreciations (depreciations) while $\Omega^{-}$and $\Omega^{+}$are the corresponding long-term pass-through coefficients; $\mathrm{W}_{\mathrm{ST}}$ and $\mathrm{W}_{\mathrm{LT}}$ are the Wald tests for short-term additive and long-term symmetric ERPT respectively and the values represent their respective F-statistics; Unr. stands for unrestricted NARDL models while Rest denotes the restricted NARDL models. The results of the cointegration analysis allows us to perform the Wald tests using the unrestricted (a) NARDL Equation (2) as a benchmark model and running the restricted ${ }^{(b)}$ NARDL Equation (3) and ${ }^{(c)}$ NARDL Equation (4) in the suitable case following the general-to-specific approach (uni-directional method and $p$-value backwards $10 \%$ significance level as stopping criteria) with a maximum lag 4 . The symbols ***,** and * show the significance (rejection of null hypothesis $\mathrm{H}_{0}$ ) at the $10 \%, 5 \%$ and $1 \%$ levels respectively. Authors' calculations using Eviews 9 .

Then, co-integration analysis and symmetry tests were implemented by using the different NARDL models to examine the long-term relationship between variables and determine suitable model specifications for each country.

Table 7. Symmetry tests for the other Sub-Saharan African Countries.

\begin{tabular}{|c|c|c|c|}
\hline Country & $\begin{array}{c}\text { Short-Term Tests }\left(\mathrm{W}_{\mathrm{ST}}\right): \\
\boldsymbol{H}_{0}: \sum_{k}^{s} \boldsymbol{\theta}_{i, k}^{-}=\sum_{k}^{t} \theta_{i, k}^{+}\end{array}$ & $\begin{array}{c}\text { Long-Term Tests }\left(\mathrm{W}_{\mathrm{LT}}\right) \text { : } \\
H_{0}: \Omega^{-}=\Omega^{+}\end{array}$ & $\begin{array}{l}\text { Unrestricted \& Restricted } \\
\text { NARDL Models }\end{array}$ \\
\hline \multicolumn{4}{|l|}{ Other SSA $\left(K_{O}=26\right)$} \\
\hline Angola (c) & - & $5.268^{* *}$ & Rest. NARDL model (4) \\
\hline Botswana (a) & - & 12.604 * & Rest. NARDL model (4) \\
\hline Cabo Verde ${ }^{(a)}$ & 0.000 & 1.578 & Rest. NARDL model (5) \\
\hline Comoros ${ }^{(a)}$ & 10.231 * & 19.186 * & Unr. NARDL model (2) \\
\hline Congo, Dem. Rep. (a) & $89.649 *$ & $18.103 *$ & Unr. NARDL model (2) \\
\hline Ethiopia (a) & $4.735^{* *}$ & 2.021 & Rest. NARDL model (3) \\
\hline Gambia $^{(a)}$ & $3.386^{* * *}$ & $31.198 *$ & Unr. NARDL model (2) \\
\hline Ghana $^{(a)}$ & $6.008^{* *}$ & 0.201 & Rest. NARDL model (3) \\
\hline Guinea (a) & $0.533 *$ & $9.784 *$ & Unr. NARDL model (2) \\
\hline Kenya ${ }^{\text {(a) }}$ & 1.749 & $55.533 *$ & Rest. NARDL model (4) \\
\hline Lesotho $^{(a)}$ & $6.786^{* *}$ & 0.046 & Rest. NARDL model (3) \\
\hline Liberia ${ }^{(a)}$ & 1.336 & $15.238 *$ & Rest. NARDL model (4) \\
\hline Madagascar (a) & $15.562 *$ & $10.967 *$ & Unr. NARDL model (2) \\
\hline Malawi (b) & $3.007^{* * *}$ & - & Rest. NARDL model (3) \\
\hline Mauritius (a) & 0.022 & $52.450 *$ & Rest. NARDL model (4) \\
\hline Mozambique $^{(a)}$ & $5.109^{* * *}$ & 0.017 & Rest. NARDL model (3) \\
\hline Namibia ${ }^{\text {(a) }}$ & 1.117 & $31.412 *$ & Rest. NARDL model (4) \\
\hline Nigeria ${ }^{(a)}$ & 17.208 * & $482.010 *$ & Unr. NARDL model (2) \\
\hline Rwanda (a) & $8.007 *$ & 2.303 & Rest. NARDL model (3) \\
\hline Sao Tome \& Prin. ${ }^{(a)}$ & $9.692 *$ & 0.001 & Rest. NARDL model (3) \\
\hline Seychelles (c) & - & $97.620 *$ & Rest NARDL model (4) \\
\hline South Africa (a) & $5.793 * *$ & $23.204 *$ & Unr. NARDL model (2) \\
\hline Swaziland ${ }^{(a)}$ & - & $3.777^{* * *}$ & Rest. NARDL model (4) \\
\hline Tanzania ${ }^{(a)}$ & 1.086 & $5.463 * *$ & Rest. NARDL model (4) \\
\hline Uganda ${ }^{(c)}$ & - & $90.710 *$ & Rest. NARDL model (4) \\
\hline Zambia (a) & 1.479 & $4.405 * *$ & Rest. NARDL model (4) \\
\hline
\end{tabular}


The hypothesis $\left(H_{1}^{0}\right)$ of a long-term symmetrical exchange rate pass-through (ERPT) is rejected in 30 out of 40 SSA countries. Therefore, the evidence of a long-term asymmetrical ERPT is strongly confirmed in most of the SSA countries, 12 of them in the CFA franc zone.

Concerning the short-term analysis, the similar hypothesis $\left(H_{4}^{0}\right)$ of symmetry does not hold (i.e., is rejected) in 24 countries. Thus, we found many cases of short- and long-term asymmetries across the SSA countries, denoting that the exchange rate movements (positive versus negative changes) do not have the same impact on domestic prices in that region except for Cabo Verde, where the symmetry hypothesis cannot be rejected in either the short or long term. As a result of these findings, the last column of Tables 6 and 7 shows the appropriate NARDL model retained to estimate the pass-through for each country. Accordingly, Tables 8 and 9 present the results of the asymmetrical ERPT compared with those in the symmetrical specification.

The hypothesis $\left(H_{0}^{2}\right)$ of zero long-term pass-through is rejected in 22 countries for depreciations and 17 for appreciations, whereas the counterpart hypothesis $\left(H_{0}^{5}\right)$ of zero short-term ERPT cannot hold in 19 cases for depreciations against 16 for appreciations.

These findings reveal that not taking into account asymmetries in the relationship between exchange rates and consumer prices in the SSA region probably results in a biased conclusion with regard to the appropriate policy to be implemented.

There is a positive association between changes in consumer prices and local currency depreciations, contrary to appreciations, in most of the SSA countries, especially in the CFA franc zone (WAEMU and CEMAC), over the long term. In fact, the positive association between depreciations and local prices is expected, because weakening of the domestic currency makes imports more expensive, leading to increased consumer prices, all other things being unchanged. Another finding is that consumer prices react more strongly and significantly to local currency depreciations than appreciations in several SSA countries over the sample period, since the pass-through coefficients for appreciations are smaller than those for depreciations in absolute value $\left(\left|\Omega^{-}\right| \prec\left|\Omega^{+}\right|\right.$and $\left.\left|\sum_{i}^{s} \theta^{-}\right| \prec\left|\sum_{i}^{s} \theta^{+}\right|\right)$, especially in the CFA Franc zone.

Furthermore, the hypotheses $\left(H_{0}^{3}\right)$ and $\left(H_{0}^{6}\right)$ of complete exchange rate pass-through (ERPT) into consumer prices are rejected in all but nine of the SSA countries; evidence of complete ERPT is found only for depreciations in Angola and Kenya and in one case for appreciation in Liberia, as well as a symmetrical ERPT in Ghana and Lesotho over the long term and only one case in the short term for depreciation in Mozambique (results are available upon request). Thus, a 1\% depreciation of Angolan kwanza (Kz) (with respect to Kenyan shilling, KES) induces a significant increase of $0.93 \%$ (with respect to $0.67 \%$ ) that is fully transmitted to consumer prices in Angola (respective to Kenya) in the long term.

However, a $1 \%$ appreciation of LRD increases prices significantly by $0.77 \%$ in Liberia over the long term. Generally, the exchange rate movements are not fully transmitted to consumer prices in most SSA countries, and this can be due to some improvements in macroeconomic policies.

Additionally, the adjustment speed of consumer prices toward long-term equilibrium prices is higher in the asymmetrical specifications (NARDL Equations (2)-(4)) compared with the linear specification (Equation (5)), where the asymmetries are neglected. The pass-through coefficients are greater in the symmetrical (linear) model of Equation (5) than in the asymmetrical models of Equations (2)-(4), especially over the long term in the CFA franc zone, whereas there is mixed evidence in the other SSA countries. Nevertheless, there is a positive relationship between consumer prices and exchange rate changes over time in the linear specification (Equation (5)), whereas this positive relationship over time cannot hold when allowing for asymmetries. 
Table 8. Exchange Rate Pass-Through (ERPT) for CFA Franc Zone.

\begin{tabular}{|c|c|c|c|c|c|c|c|c|c|c|c|c|c|c|}
\hline \multirow[b]{3}{*}{ Countries } & \multicolumn{9}{|c|}{ Asymmetric Exchange Rate Pass-Through (ERPT) } & \multicolumn{5}{|c|}{ Symmetric Exchange Rate Pass-Through (ERPT) } \\
\hline & \multicolumn{4}{|c|}{ Long-Term ERPT } & \multicolumn{3}{|c|}{ Short-Term ERPT } & \multicolumn{2}{|c|}{ Diagnostics } & \multicolumn{2}{|c|}{ Long-Term ERPT } & \multirow{2}{*}{$\begin{array}{c}\begin{array}{c}\text { Short-Term } \\
\text { ERPT }\end{array} \\
\mathbf{S}_{\mathrm{T}} \mathrm{S} \\
\sum_{k}^{s} \theta_{i, k}\end{array}$} & \multicolumn{2}{|c|}{ Diagnostics } \\
\hline & $\begin{array}{c}\text { Adj. } \\
\text { Speed } \beta_{i}\end{array}$ & $\begin{array}{c}\text { Appr(-) } \\
\Omega_{i}^{-}\end{array}$ & $\begin{array}{c}\operatorname{Depr}(+) \\
\Omega_{i}^{+}\end{array}$ & $\begin{array}{c}\mathrm{L}_{\mathrm{T}} \mathrm{S} \\
\Omega_{i}\end{array}$ & $\begin{array}{c}\text { Appr(-) } \\
\sum_{k}^{s} \theta_{i, k}^{-}\end{array}$ & $\begin{array}{c}\text { Depr(+) } \\
\sum_{k}^{s} \theta_{i, k}^{+}\end{array}$ & $\begin{array}{l}\mathbf{S}_{\mathrm{T}} \mathbf{S} \\
\sum_{k}^{s} \theta_{i, k}\end{array}$ & $\overline{R^{2}}$ & $x^{2} s c$ & $\underset{\text { Speed } \Phi_{i}}{\text { Adj. }}$ & $\begin{array}{c}\mathbf{L}_{\mathrm{T}} \mathrm{S} \\
\Omega_{i}\end{array}$ & & $\overline{R^{2}}$ & $\chi^{2} \mathrm{sc}$ \\
\hline \multicolumn{15}{|l|}{ WAEMU $\left(K_{W}=8\right)$} \\
\hline Benin & $-0.072 * * *$ & -0.034 & 0.282 & & - & 0.347 * & & 0.638 & 0.459 & -0.025 & -0.711 & $0.131 *$ & 0.655 & 0.157 \\
\hline Burkina Faso & -0.194 * & $0.251 * *$ & $0.396 *$ & & - & $0.166^{*}$ & & 0.545 & $2.706^{* * *}$ & -0.154 * & $0.463 *$ & $0.173 *$ & 0.467 & 1.823 \\
\hline Cote d'Ivoire & -0.264 * & $0.207^{*}$ & 0.455 * & & - & - & 0.184 * & 0.635 & 0.401 & -0.029 & 0.353 & $0.219 *$ & 0.591 & $3.332 * *$ \\
\hline Guinea-Bissau & $-0.118 *$ & - & - & $0.990 *$ & - & $-0.643 *$ & & 0.599 & 0.404 & $-0.096^{*}$ & $1.151 *$ & $-0.218^{* *}$ & 0.548 & 0.905 \\
\hline Mali & $-0.322 *$ & $0.122 * *$ & $0.356^{*}$ & & $0.176^{*}$ & 0.011 & & 0.658 & 0.368 & $-0.061^{* *}$ & 0.426 * & 0.180 * & 0.599 & 0.304 \\
\hline Niger & $-0.277^{*}$ & 0.132 & 0.363 * & & - & - & $0.272 *$ & 0.688 & 0.881 & -0.218 * & $0.699 *$ & $0.162 *$ & 0.650 & 0.243 \\
\hline Senegal & $-0.085^{* * *}$ & -0.372 & 0.019 & & - & - & -0.011 & 0.624 & 0.937 & -0.038 & -0.074 & -0.010 & 0.622 & 1.186 \\
\hline Togo & $-0.188 *$ & -0.508 & 0.045 & & - & - & $1.310 *$ & 0.632 & 0.200 & $-0.142 *$ & $0.641 *$ & $0.739 *$ & 0.585 & 0.759 \\
\hline \multicolumn{15}{|l|}{ CEMAC $\left(K_{C}=6\right)$} \\
\hline Cameroon & $-0.165^{*}$ & $-0.474^{*}$ & 0.077 & & $-0.142 *$ & $0.472 *$ & & 0.854 & 0.831 & $-0.036^{* * *}$ & 0.090 & 0.367 * & 0.720 & 4.074 \\
\hline Cent. African R. & 0.019 & - & - & -0.088 & $-0.200 * *$ & $0.325 *$ & & 0.583 & 0.131 & 0.007 & 0.295 & $0.117^{*}$ & 0.516 & 0.273 \\
\hline Chad & $-0.458^{*}$ & $0.390^{*}$ & 0.601 * & & $-0.249 * *$ & 0.447 * & & 0.616 & 0.646 & $-0.290 *$ & $0.708 *$ & $0.260 *$ & 0.555 & 0.139 \\
\hline Congo Rep. & $-0.161 *$ & -0.105 & 0.351 & & 0.517 * & 0.164 * & & 0.721 & 1.805 & -0.051 & $0.479 * * *$ & $0.162 *$ & 0.576 & 0.864 \\
\hline Eq. Guinea & $-0.224 *$ & 0.035 & $0.518 *$ & & -0.123 & $0.216^{*}$ & & 0.748 & 0.375 & -0.026 & 0.530 & $0.289 *$ & 0.580 & 0.897 \\
\hline Gabon & $-0.175^{*}$ & -0.070 & 0.317 * & & $-0.311 *$ & 0.304 * & & 0.725 & 0.778 & $-0.097 *$ & $0.404 *$ & -0.005 & 0.615 & 0.250 \\
\hline
\end{tabular}

Note: $\beta_{i}$ and $\Phi_{i}$ are the speeds of adjustment to the long-term equilibrium in the nonlinear NARDLs (models (2), (3) and (4)) and the linear model (5), respectively; $\bar{R}^{2}$ is the adjusted

$\mathrm{R}$-squared and $\chi^{2} \mathrm{sc}$, the Breusch-Godfrey serial correlation LM test. The models have been estimated following the general-to-specific approach with a maximum lag 4 . $\sum_{k}^{s} \theta_{i, k}^{-}\left(\sum_{k}^{t} \theta_{i, k}^{+}\right)$is the short-term additive pass-through for appreciations (depreciations) while $\Omega^{-}$and $\Omega^{+}$are their corresponding long-term pass-through coefficients. K is the number of the panel units. The symbols ${ }^{* * *}, * *$ and ${ }^{*}$ show the significance at the $10 \%, 5 \%$ and $1 \%$ levels respectively. Authors' calculations using Eviews 9. 
Table 9. Exchange Rate Pass-Through (ERPT) in the other sub-Saharan African Countries.

\begin{tabular}{|c|c|c|c|c|c|c|c|c|c|c|c|c|c|c|}
\hline \multirow[b]{3}{*}{ Countries } & \multicolumn{9}{|c|}{ Asymmetric Exchange Rate Pass-Through (ERPT) } & \multicolumn{5}{|c|}{ Symmetric Exchange Rate Pass-Through (ERPT) } \\
\hline & \multicolumn{4}{|c|}{ Long-Term ERPT } & \multicolumn{3}{|c|}{ Short-Term ERPT } & \multicolumn{2}{|c|}{ Diagnostics } & \multicolumn{2}{|c|}{ Long-Term ERPT } & \multirow{2}{*}{$\begin{array}{c}\begin{array}{c}\text { Short-Term } \\
\text { ERPT }\end{array} \\
\mathrm{S}_{\mathrm{T}} \mathrm{S} \\
\sum_{k}^{s} \theta_{i, k} \\
\end{array}$} & \multicolumn{2}{|c|}{ Diagnostics } \\
\hline & $\begin{array}{c}\text { Adj. } \\
\text { Speed } \beta_{i}\end{array}$ & $\begin{array}{c}\text { Appr(-) } \\
\Omega_{i}^{-}\end{array}$ & $\begin{array}{c}\operatorname{Depr}(+) \\
\Omega_{i}^{+}\end{array}$ & $\begin{array}{c}\mathrm{L}_{\mathrm{T}} \mathrm{S} \\
\Omega_{i}\end{array}$ & $\begin{array}{c}\text { Appr(-) } \\
\sum_{k}^{s} \theta_{i, k}^{-}\end{array}$ & $\begin{array}{c}\text { Depr(+) } \\
\sum_{k}^{s} \theta_{i, k}^{+}\end{array}$ & $\begin{array}{l}\mathrm{S}_{\mathrm{T}} \mathrm{S} \\
\sum_{k}^{s} \theta_{i, k}\end{array}$ & $\overline{R^{2}}$ & $x^{2} \mathrm{sC}$ & $\underset{\text { Speed } \Phi_{i}}{\text { Adj. }}$ & $\begin{array}{c}\mathrm{L}_{\mathrm{T}} \mathrm{S} \\
\Omega_{i}\end{array}$ & & $\overline{R^{2}}$ & $x^{2} s c$ \\
\hline Other SSA $\left(K_{O}=26\right)$ & & & & & & & & & & & & & & \\
\hline Angola & -0.072 * & $-4.121 * * *$ & $0.934 *$ & - & - & - & $0.506 *$ & 0.938 & 0.084 & -0.034 & $0.878 *$ & $0.562 *$ & 0.935 & 0.570 \\
\hline Botswana & -0.021 & -0.748 & -0.129 & - & - & - & - & 0.601 & 0.529 & -0.015 & -0.607 & - & 0.598 & 0.782 \\
\hline Cabo Verde & - & - & - & - & - & - & - & - & - & -0.134 * & $0.183 * * *$ & $0.105^{* *}$ & 0.320 & 1.182 \\
\hline Comoros & -0.859 * & -0.830 * & -0.356 * & - & 0.272 & $-1.265 *$ & - & 0.852 & $3.987^{* *}$ & $-0.872 *$ & $-0.466^{*}$ & $0.913 *$ & 0.584 & 0.242 \\
\hline Congo, Dem. Rep. & -0.207 * & -1.784 * & 0.719 * & - & 4.400 * & $-0.089 * * *$ & - & 0.901 & 0.799 & $0.142^{* * *}$ & $1.075 *$ & 1.010 * & 0.781 & 0.766 \\
\hline Ethiopia & 0.144 * & - & - & $0.556 *$ & $-5.978 * *$ & $-0.232 *$ & $0.272 *$ & 0.626 & 1.554 & $-0.133 * * *$ & 0.131 & $-0.114^{* *}$ & 0.626 & 2.234 \\
\hline Gambia & -0.172 * & 0.038 & $0.399 *$ & - & $-0.069^{* * *}$ & - & - & 0.354 & 1.127 & -0.058 * & $0.826^{*}$ & - & 0.183 & 0.283 \\
\hline Ghana & $-0.056^{* *}$ & - & - & $0.853 *$ & $-0.573^{* * *}$ & $0.246^{*}$ & - & 0.622 & $2.576^{* * *}$ & -0.044 & $0.871 *$ & $0.251 *$ & 0.617 & $2.767^{* * *}$ \\
\hline Guinea & 0.169 * & $1.703 *$ & $2.172 *$ & - & 0.533 * & -0.000 & - & 0.938 & $3.238^{* * *}$ & 0.032 & 3.597 & 0.193 * & 0.656 & $2.792^{* * *}$ \\
\hline Kenya & $0.096 *$ & $-1.556 *$ & $0.674^{* *}$ & - & - & - & - & 0.476 & 0.127 & -0.009 & -5.014 & $0.247 *$ & 0.471 & 1.173 \\
\hline Lesotho & -0.015 & - & - & -0.887 & $-0.125^{* * *}$ & 0.164 * & - & 0.415 & 0.973 & $-0.027^{* *}$ & -0.324 & - & 0.373 & 1.673 \\
\hline Liberia & -0.230 * & 0.771 * & $1.549 *$ & - & -0.193 * & $0.205^{\text {** }}$ & - & 0.645 & 1.362 & 0.033 & 1.634 & $0.425^{* *}$ & 0.481 & 1.253 \\
\hline Madagascar & -0.161 * & 0.149 & $0.699 *$ & - & - & - & - & 0.426 & 0.578 & -0.071 * & $0.806 *$ & - & 0.410 & 1.067 \\
\hline Malawi & -0.108 * & - & - & 0.851 * & 0.276 & $0.102 *$ & - & 0.860 & 1.455 & $-0.077^{* *}$ & 0.830 * & 0.172 & 0.862 & 1.296 \\
\hline Mauritius & -2.751 * & $0.226^{* *}$ & 0.765 * & - & - & - & -0.135 * & 0.222 & 0.359 & $-0.020^{* * *}$ & 0.618 & -0.121 * & 0.201 & 0.672 \\
\hline Mozambique & -0.643 * & - & - & $0.747 *$ & $0.747^{* * *}$ & -0.558 * & - & 0.932 & $6.233^{* *}$ & 0.060 & 4.137 & 0.995 * & 0.894 & $4.724 * *$ \\
\hline Namibia & $-0.118^{* * *}$ & -1.067 & 0.011 & - & - & - & 0.082 & 0.349 & 0.654 & -0.013 & 0.642 & 0.047 & 0.082 & 0.265 \\
\hline Nigeria & -0.235 * & $-5.750 *$ & $0.561 *$ & - & $2.625 *$ & 0.107 * & - & 0.713 & 0.072 & -0.021 ** & 0.316 & $0.040^{* *}$ & 0.630 & 0.843 \\
\hline Rwanda & $-0.086^{*}$ & - & - & 0.655 * & - & - & - & 0.264 & 2.114 & $-0.086^{*}$ & 0.655 * & - & 0.264 & 2.114 \\
\hline Sao Tome and Prin & $-0.126^{*}$ & - & - & $1.878 *$ & $0.379 *$ & -0.140 & - & 0.700 & 1.135 & -0.209 * & $1.983 *$ & -0.137 & 0.690 & 0.975 \\
\hline Seychelles & -0.526 * & $0.302 *$ & $0.632 *$ & - & - & - & $0.347 *$ & 0.606 & $4.575^{* *}$ & -0.243 * & 0.974 * & 0.221 * & 0.527 & 0.764 \\
\hline South Africa & -0.050 * & 0.103 & $0.416^{*}$ & - & - & -0.055 ** & - & 0.536 & 1.121 & -0.012 & -0.033 & 0.004 & 0.522 & 0.857 \\
\hline Swaziland & $-0.061^{* * *}$ & 0.257 & $0.595 *$ & - & - & - & - & 0.458 & 0.184 & $-0.030 * * *$ & 0.714 * & - & 0.456 & 0.170 \\
\hline Tanzania & -0.125 * & $-1.554^{* * *}$ & 0.368 & - & - & - & -0.254 * & 0.546 & 1.892 & 0.014 & 5.901 & 0.438 * & 0.524 & 1.320 \\
\hline Uganda & $-0.277^{*}$ & -0.801 * & $0.238^{* *}$ & - & - & - & $0.278 *$ & 0.295 & 1.667 & -0.040 & $0.888^{* *}$ & $0.085^{* *}$ & 0.223 & $4.131^{* *}$ \\
\hline Zambia & $-0.125 *$ & 0.547 * & 0.775 * & - & - & - & $0.114 * *$ & 0.749 & 0.260 & $-0.065^{* *}$ & 0.658 * & $0.173 *$ & 0.754 & 1.454 \\
\hline
\end{tabular}

Note: see Table 8. 
Finally, we computed the dynamic multipliers for each country to trace the patterns of the exchange rate pass-through into prices over time (see Appendix A, Figures A1 and A2). Figures A1 and A2 present the patterns of the dynamic multipliers in the CFA franc zone (WAEMU and CEMAC) and the other SSA countries, where the asymmetry line shows how consumer prices react differently to shocks to exchange rates during periods of depreciation and appreciation.

These figures illustrate that consumer prices respond more strongly to exchange rate depreciations than appreciations in the very short term (after a few quarters) in several SSA countries, but the asymmetrical pass-through pattern still increases even in the very short term and tends toward zero, particularly in Cabo Verde, justifying the symmetrical pass-through in this country.

\subsection{Results of Dynamic Panel ERPT Estimations}

We aggregated the countries into two main subgroups: the CFA franc zone (WAEMU and CEMAC subregions) and the other SSA countries (with a floating exchange rate regime) that do not belong to the CFA franc zone, which has a fixed exchange rate regime and almost similar currency.

\subsubsection{Robustness Tests under Cross-Sectional Dependence Analysis}

We began the robustness tests by performing four cross-sectional dependence tests, as mentioned in Section 3, i.e., the $R_{\text {ave }}$ statistic of Friedman (1937), the $C D_{L M}$ of Breusch and Adrian (1980), the FT statistic of Frees (1995), and the CD test of Pesaran (2004), under the null hypothesis of no autocorrelation of the residuals across panel units. Table 10 presents the results of the tests and their corresponding $p$-Values after running fixed and random effects estimations. The results confirm the existence of cross-sectional dependence between countries, since the $p$-values of these tests are less than conventional significance levels $(1 \%, 5 \%$, or $10 \%)$ and lead to rejection of the null hypothesis.

Table 10. Cross-sectional dependence tests.

\begin{tabular}{ccccccccc}
\hline Cross Dependence Tests & \multicolumn{2}{c}{$C D$} & \multicolumn{2}{c}{$\boldsymbol{F T}$} & \multicolumn{2}{c}{$\boldsymbol{R}_{\text {ave }}$} & \multicolumn{2}{c}{$C \boldsymbol{D}_{\boldsymbol{L M}}$} \\
\cline { 2 - 7 } & Coef & $\boldsymbol{p}$-Value & Coef & $\boldsymbol{p}$-Value & Coef & $\boldsymbol{p}$-Value & Coef & $\boldsymbol{p}$-Value \\
\hline Fixed effect (WAEMU) & 24.262 & 0.000 & 3.368 & 0.000 & 519.615 & 0.000 & 1559.421 & 0.000 \\
Random effect (WAEMU) & 24.116 & 0.000 & 3.409 & 0.000 & 528.953 & 0.000 & 1553.25 & 0.000 \\
Fixed effect (CEMAC) & 6.21 & 0.000 & 1.207 & 0.000 & 165.836 & 0.000 & 314.531 & 0.000 \\
Random effect (CEMAC) & 5.787 & 0.000 & 0.982 & 0.000 & 180.469 & 0.000 & 271.852 & 0.000 \\
Fixed effect (Other countries) & 25.842 & 0.000 & 7.046 & 0.000 & 162.692 & 0.000 & 5647.464 & 0.000 \\
Random effect (Other countries) & 13.113 & 0.000 & 7.704 & 0.000 & 159.692 & 0.000 & 8331.561 & 0.000 \\
Fixed effect (All countries) & 61.443 & 0.000 & 12.345 & 0.000 & 423.375 & 0.000 & $18,037.34$ & 0.000 \\
Random effect (All countries) & 29.254 & 0.000 & 9.441 & 0.000 & 186.45 & 0.000 & $23,452.55$ & 0.000 \\
\hline
\end{tabular}

Note: $C D, F T, R_{\text {ave }}$ and $C D_{L M}$ are the Pesaran (2004), Frees (1995), Friedman (1937) and Breusch and Adrian (1980) test statistics respectively. Authors' calculations using Stata 14.

\subsubsection{Results of Panel Pass-Through Estimations}

We performed robustness tests through two dynamic panel models accounting for cross-sectional dependence, autocorrelations, and heteroscedasticity: the feasible generalized least squares (FGLS) of Parks (1967) and the pooled OLS regression using robust standard errors of Driscoll and Kraay (1998). The two estimators were employed to check whether our findings were still robust, and the results are depicted in Table 11. 
Table 11. Panel ERPT Estimation under Cross-Sectional Dependence Analysis.

\begin{tabular}{|c|c|c|c|c|c|c|c|c|c|c|}
\hline \multirow[b]{3}{*}{ Country } & \multicolumn{6}{|c|}{ Long-Term Relationship } & \multicolumn{4}{|c|}{ Short-Term Relationship } \\
\hline & \multirow[b]{2}{*}{$\begin{array}{c}\text { Asym. } \\
\text { Adjust. } \\
\text { Speed, } \beta_{i}\end{array}$} & \multirow[b]{2}{*}{$\begin{array}{c}\text { Sym. } \\
\text { Adjust. } \\
\text { Speed, } \Phi_{i}\end{array}$} & \multicolumn{3}{|c|}{ Long-Term ERPT } & \multirow[b]{2}{*}{$\begin{array}{c}\text { Wald Test } \\
\text { Long-Term } \\
H_{0}: \Omega^{-}=\Omega^{+}\end{array}$} & \multicolumn{3}{|c|}{ Cumulative ERPT } & \multirow{2}{*}{$\begin{array}{c}\text { Wald Test } \\
\text { Cumulative } \\
H_{0}: \sum_{k} \theta_{i, k}^{-}=\sum_{k}^{t} \theta_{i, l}^{+}\end{array}$} \\
\hline & & & $\begin{array}{c}\text { Appr(-): } \\
\Omega_{i}^{-}\end{array}$ & $\begin{array}{c}\text { Depr(+): } \\
\Omega_{i}^{+}\end{array}$ & $\mathrm{LT}_{\mathrm{ERPT}} \boldsymbol{\Omega}_{i}$ & & $\begin{array}{c}\text { Appr(-) } \\
\sum_{k}^{s} \theta_{i, k}^{-}\end{array}$ & $\begin{array}{c}\text { Depr(+) } \\
\sum_{k}^{t} \theta_{i, k}^{+}\end{array}$ & $\begin{array}{c}\mathrm{ST}_{\mathrm{ERPT}} \\
\sum_{k}^{t} \theta_{i, k}\end{array}$ & \\
\hline \multicolumn{11}{|l|}{ FGLS estimator } \\
\hline CFA Franc zone $(K=14)$ & $-0.049 *$ & $-0.046 *$ & $0.447^{*}$ & $0.637^{*}$ & $0.648^{*}$ & $16.530 *$ & $-0.118^{*}$ & $0.265^{*}$ & $0.183 *$ & $106.530 *$ \\
\hline WAEMU $\left(K_{W}=08\right)$ & $-0.064 *$ & $-0.058^{*}$ & $0.387^{*}$ & $0.544^{*}$ & $0.563 *$ & 11.410 * & $-0.115^{*}$ & $0.258^{*}$ & $0.177^{*}$ & $60.240 *$ \\
\hline CEMAC $\left(K_{C}=06\right)$ & $-0.033^{*}$ & $-0.032 *$ & $0.574^{* *}$ & $0.845^{*}$ & $0.839 *$ & $4.860 * *$ & $-0.130 *$ & $0.277^{*}$ & 0.187 * & $46.840 *$ \\
\hline Other SSA $\left(K_{O}=26\right)$ & $-0.016^{*}$ & $-0.016^{*}$ & $0.410 *$ & $0.195 *$ & $0.032 * *$ & $8.290^{*}$ & -0.012 & $0.118^{*}$ & $0.087 *$ & $29.350 *$ \\
\hline All SSA countries $(K=40)$ & $-0.015^{*}$ & $-0.015^{*}$ & $0.420 *$ & $0.244^{*}$ & 0.011 & $9.570 *$ & $-0.030^{* *}$ & $0.176^{*}$ & $0.127^{*}$ & $114.440 *$ \\
\hline \multicolumn{11}{|l|}{ POLS_DK estimator } \\
\hline CFA Franc zone $(\mathrm{K}=14)$ & $-0.050 *$ & $-0.049 *$ & $0.438 * *$ & $0.640 *$ & $0.673 *$ & $6.290 * *$ & -0.091 & $0.244^{*}$ & $0.175^{*}$ & $10.220 *$ \\
\hline WAEMU $\left(\mathrm{K}_{\mathrm{W}}=08\right)$ & $-0.057 *$ & $-0.056^{*}$ & $0.401^{* *}$ & $0.553 *$ & $0.581 *$ & $3.480 * * *$ & -0.058 & $0.222 *$ & $0.165^{*}$ & $6.040 * *$ \\
\hline CEMAC $\left(K_{C}=06\right)$ & $-0.038^{*}$ & $-0.036^{*}$ & $0.552^{* * *}$ & $0.858^{*}$ & $0.878^{*}$ & $5.040 * *$ & $-0.147^{* *}$ & $0.283 *$ & $0.193 *$ & $16280 *$ \\
\hline Other SSA $\left(\mathrm{K}_{\mathrm{O}}=26\right)$ & $-0.020 *$ & $-0.018 *$ & 0.181 & $0.125^{*}$ & $0.040 *$ & 0.270 & 0.105 & $0.196^{*}$ & $0.187^{*}$ & 1.520 \\
\hline All SSA countries $(K=40)$ & $-0.020 *$ & $-0.019 *$ & $0.174^{* * *}$ & $0.140 *$ & 0.027 & 0.130 & 0.057 & 0.210 * & $0.191 *$ & $7.460^{*}$ \\
\hline
\end{tabular}

Note: $\beta_{i}$ and $\Phi_{i}$ represent the speed of adjustment in the nonlinear model (2) and the linear model (5), respectively. FGLS stands for the feasible generalized least squares; POLS_DK denotes the pooled OLS (ordinary least squares) estimator using Driscoll-Kraay standard errors which are robust to heteroskedasticity, autocorrelation and the cross-sectional dependence of errors up to lag 4 between the panel units. The numbers in italic indicate complete ERPT, whereas the statistics for Wald tests are chi-squared values. The symbols ***,** and * show the significance at $10 \%, 5 \%$ and $1 \%$ levels respectively. Authors' calculations using Stata 14 
We still found that the hypotheses $\left(H_{0}^{1}\right)$ and $\left(H_{0}^{4}\right)$ of a symmetrical ERPT over the short and long term are rejected in the CFA franc zone (WAEMU and CEMAC subregions), while this is not the case for the other SSA countries and the entire SSA region over the long term according to the pooled OLS regression using robust standard errors of Driscoll and Kraay (1998). Accordingly, the FGLS and pooled OLS estimators both provide evidence of asymmetrical behavior of exchange rates into consumer prices in all subregions as well as the entire SSA region over the short term. These two estimators validate our findings of non-zero pass-through for depreciations and appreciations (rejection of Hypotheses 2 and 5) as well as the linear ARDL model of Equation (5) over the short and long term. Moreover, the results of the panel analysis reject the hypotheses $\left(H_{0}^{3}\right)$ and $\left(H_{0}^{6}\right)$ of a complete ERPT for appreciations and depreciations, respectively, over the long and short term, except for the CEMAC subregion, where both FGLS and pooled OLS estimators reveal a complete ERPT for local currency depreciations and appreciations in the long term (results are available upon request).

These estimators induce more accurate, consistent estimates and validate the finding that exchange rates are more strongly passed through to prices during depreciations than appreciations, especially in the CFA franc zone in the long term. In addition, the evidence that consumer prices react more strongly to local currency depreciations than appreciations is largely valid across subregions as well as in the entire SSA region during the short term. Therefore, our results of asymmetric exchange rate pass-through (ERPT) are more robust during the short and long term for the global CFA franc zone, while these findings are significantly appropriate in the short term for the other SSA countries and the entire SSA region.

The evidence for incomplete ERPT is robust in the SSA countries over the short and long term. In addition, consumer prices are negatively associated with local currency appreciations but positively linked to depreciations over the short term, whereas they have a positive effect and different impacts on consumer prices in the long term. For instance, both FGLS and pooled OLS estimates reveal that a $10 \%$ depreciation of the exchange rate leads to about a $6 \%$ increase in consumer prices in the CFA franc zone, while the same percentage causes prices to rise by $4 \%$ or so following appreciations in the long term, with different magnitudes across WAEMU and CEMAC subregions. In the other SSA countries and the entire region, $10 \%$ depreciations (appreciations) increase price levels by approximately $2 \%$ over the long term. At the same time, local prices decrease by approximately $1 \%$ (respectively about $0.1 \%$ in the other SSA countries and around $0.3 \%$ in the region) with a $10 \%$ appreciation of the exchange rate in the CFA franc zone according to FGLS, whereas 10\% depreciation of the local currency raises consumer prices above $2 \%$ (less than $2 \%$ for the other SSA economies, as well as the region) during the short term.

In short, the evidence of an asymmetrical exchange rate pass-through in most of the SSA countries nourishes the thought that producers may not distinguish the diverse effects of local currency depreciations and appreciations on domestic prices, or they may be unable or reluctant to consider these effects in their operating margins over the short term. This situation may also encourage alternative speculation in the short term, since local currency depreciations and appreciations do not have the same impact on consumer prices. Besides, the stronger pass-through elasticities for depreciations than for appreciations reveal that foreign producers are prone to raise prices in most SSA countries to limit the decrease of profits, which is to partially pass local currency depreciations into consumer prices while keeping constant prices and raising their markups by absorbing appreciations and quoting for competitive prices.

Our findings show a lack of competitive market structures in the SSA region denoted by this kind of high pricing power, since foreign producers have a large market share in this region. For instance, the shares of European and Asian exports in world merchandise exports to Africa are about $34.7 \%$ and $32.4 \%$, respectively, while only $15.4 \%$ came from Africa in $2014 .^{1}$

1 World Trade Organization, International Trade Statistics 2015. 
Furthermore, we find that the exchange rate pass-through is high in the CFA franc region, which has a relatively low inflationary environment as measured by the average inflation rate $(1.04 \%)$ compared with the other SSA countries (3.13\%) associated with high inflation levels (see Table 11 ). This evidence contrasts with the Taylor (2000) hypothesis that lower ERPT is positively associated with a low inflationary environment, which reduces the pricing power of producers. In fact, the FGLS of Parks (1967) and the pooled OLS regression with standard errors of Driscoll and Kraay (1998) correct any bias resulting from the presence of cross-sectional dependence between countries.

This result points out the lack of or limited credibility of monetary policy in the SSA region and the poor transmission of its monetary policy associated with smaller financial depth struggling to adapt to exchange rate shocks. Hence, producers tend to pass through exchange rate changes when inflation expectations are not well anchored on a target, which can be interpreted as a lack of transparency in monetary policy, hindering the decline in the exchange rate pass-through into consumer prices.

In general, the results of this paper are contrary to the findings of Akofio-Sowah (2009), exhibiting declining and symmetric pass-through elasticities in 15 SSA countries under a lower inflationary environment. However, our findings are similar to those reported in the IMF's working papers (International Monetary Fund 2012) in the sense of incomplete pass-through into consumer prices, which is larger during exchange rate depreciations than appreciations over the short-term, especially in the CFA Franc zone.

\subsubsection{Estimations of Pass-Through with Respect to Size and Direction of Exchange Rates}

In this section, we decompose exchange rate movements into large and small changes in order to examine their impact on consumer prices. In order words, we investigate whether menu costs are important in the SSA countries, i.e., whether the size of the pass-through is positively related to the size of the exchange rate. Thus, we employ the approach of Pollard and Coughlin (2004) to construct large changes of exchange rates.

To do so, we distinguish large changes from small changes of exchange rate following Pollard and Coughlin (2004) in this manner:

$$
L_{t}=1 \text { if }\left|\Delta l e r_{t}\right| \geq 3 \%, L_{t}=0 \text {, otherwise and } S_{t}=1 \text { if }\left|\Delta l e r_{t}\right| \prec 3 \%, S_{t}=0 \text {, otherwise }
$$

We use these dummy variables to construct large changes $\left(L_{t} \Delta l e r_{t}\right)$ and small changes $\left(S_{t} \Delta l e r_{t}\right)$ of exchange rates.

Then we estimate the NARDL models of Equations (2) and (5) using these variables instead of $\Delta l e r_{t}$ and allowing both the linear and nonlinear specifications. Table 12 describes the size of the exchange rate across subregions as well as in the entire SSA region.

Table 12. Behavior of the exchange rate.

\begin{tabular}{ccccccc}
\hline \multicolumn{7}{c}{$\begin{array}{c}\text { Behavior of the Exchange Rate: 1990Q1-2017Q4 } \\
\text { (Percent of Total Changes) }\end{array}$} \\
\cline { 2 - 7 } Sub-Regions & \multicolumn{2}{c}{ Overall } & \multicolumn{2}{c}{ Appreciations } & \multicolumn{2}{c}{ Depreciations } \\
\cline { 2 - 7 } & $\begin{array}{c}\text { Large } \\
\text { Change }\end{array}$ & $\begin{array}{c}\text { Small } \\
\text { Change }\end{array}$ & $\begin{array}{c}\text { Large } \\
\text { Change }\end{array}$ & $\begin{array}{c}\text { Small } \\
\text { Change }\end{array}$ & $\begin{array}{c}\text { Large } \\
\text { Change }\end{array}$ & $\begin{array}{c}\text { Small } \\
\text { Change }\end{array}$ \\
\hline CFA Franc zone $(\mathrm{K}=14)$ & 58.63 & 41.37 & 29.18 & 70.82 & 28.85 & 71.15 \\
WAEMU $\left(\mathrm{K}_{\mathrm{W}}=08\right)$ & 58.41 & 41.59 & 29.82 & 70.18 & 29.01 & 70.99 \\
CEMAC $\left(\mathrm{K}_{\mathrm{C}}=06\right)$ & 58.93 & 41.07 & 28.33 & 71.67 & 28.64 & 71.36 \\
Other SSA $\left(\mathrm{K}_{\mathrm{O}}=26\right)$ & 45.10 & 54.90 & 13.42 & 86.58 & 30.15 & 69.85 \\
All SSA countries $(\mathrm{K}=40)$ & 50.27 & 49.73 & 19.37 & 80.63 & 29.63 & 70.37 \\
\hline
\end{tabular}

Note: Authors' calculations. 
The statistics reveal that there have been larger exchange rate changes in CFA franc zone (58.63\%) than in the other SSA countries (45.10\%) following the linear specification. Considering the direction of the exchange rate, we note a longer period of small changes than large changes in exchange rate across subregions. The results of the implied ERPT are presented in Table 13. First, the results suggest asymmetrical ERPT with regard to the size of the exchange rate, where the pass-through is higher after large changes in the exchange rate than small changes.

Second, the ERPT is greater during large depreciations than large appreciations of the local currency. Consumer prices decrease at a higher level after large depreciations (positive exchange rate changes) than they increase during appreciations (negative exchange rate changes) of the local currency.

Finally, we perform an additional analysis by distinguishing the sub-Saharan African (SSA) countries suffering from hyperinflation from those with low inflation levels (see Appendix B.2 for the classification, Table A2). We group the SSA countries into two main groups: countries with high inflation levels (including hyperinflation) and countries with low inflation. There are 13 countries with average inflation above the mean $(2.33 \%)$ of the entire SSA region. These countries have been considered to have high inflation levels. From this group, seven countries have a very high average inflation level (above 3.5\%), indicating that they experience hyperinflation in line with recent inflation trends observed in these countries. The remaining 27 countries have low inflation levels (below $2.33 \%$ ). The classification of these countries is shown in the Appendix B.2. Table 14 displays the results of the ERPT coefficients considering these groups of countries.

The results reject Hypotheses 1 and $4\left(H_{0}^{1}\right.$ and $\left.H_{0}^{4}\right)$ of a symmetrical ERPT to consumer prices at a $5 \%$ level of significance in the countries with high inflation in both the short term and long term. However, there is only evidence of an asymmetrical ERPT in the short term (rejection of hypothesis $\mathrm{H}_{0}^{4}$ at the $10 \%$ level) for the countries with hyperinflation following the results of FGLS, while the results of POLS_DK suggest that the asymmetrical ERPT is only valid in the long term (rejection of hypothesis $H_{0}^{1}$ at the $5 \%$ level).

The hypotheses $H_{0}^{1}$ and $H_{0}^{4}$ of a symmetrical ERPT over the long term and short term, respectively, are rejected at the $5 \%$ level in the countries with low inflation according to the FGLS results, but the asymmetrical EPRT is only valid in the short term with regard to the results of POLS_DK at the $1 \%$ level. In addition, the ERPT's coefficients are more significant during the long term than the short term across subgroups. The coefficients are greater in the countries with low inflation levels than those with high inflation and also higher over the long term than in the short term. Consumer prices converge faster to their equilibrium levels in countries with high inflation (the speed of adjustment to the equilibrium price is around 3\%) than in the countries with low inflation (approximately $1 \%$ ). In addition, the ERPT's coefficients are lower and less significant when the analysis neglects the asymmetries and nonlinearities by considering a linear specification. This evidence reveals the importance of taking into account the asymmetries and nonlinearities in the relationship between exchange rate changes and consumer prices to obtain the accurate results necessary for the implementation of appropriate policies. 
Table 13. Pass-Through with Respect to the Size and Direction of Exchange Rates

\begin{tabular}{|c|c|c|c|c|c|c|c|c|c|c|c|}
\hline \multirow{3}{*}{ Sub-Regions } & \multicolumn{3}{|c|}{ Overall } & \multicolumn{3}{|c|}{ Appreciations } & \multicolumn{3}{|c|}{ Depreciations } & \multicolumn{2}{|c|}{ Asymmetry Tests } \\
\hline & Large & Small & $\mathbf{H}_{0}$ : & Large & Small & $\mathbf{H}_{0}$ : & Large & Small & $\mathbf{H}_{0}$ : & $\mathbf{H}_{0}$ : & $\mathrm{H}_{0}:$ \\
\hline & $\mathbf{L}$ & $S$ & $\mathbf{L}=\mathbf{S}$ & LA & SA & $\mathbf{L A}=\mathbf{S A}$ & LD & SD & $\mathrm{LD}=\mathrm{SD}$ & LA $=\mathbf{L D}$ & $\mathrm{SA}=\mathrm{SD}$ \\
\hline \multicolumn{12}{|l|}{ FGLS estimator } \\
\hline CFA Franc zone $(\mathrm{K}=14)$ & $0.102 *$ & -0.047 & $5.740^{* *}$ & -0.127 * & $-0.344 *$ & $4.460 * *$ & $0.170 *$ & $0.228^{* *}$ & 0.380 & $120.060 *$ & 11.750 * \\
\hline WAEMU $\left(K_{W}=08\right)$ & $0.101 *$ & -0.061 & $4.050 * *$ & $-0.125 *$ & $-0.289 * *$ & 1.500 & $0.169 *$ & 0.150 & 0.020 & $68.720 *$ & $4.100 * *$ \\
\hline CEMAC $\left(K_{C}=06\right)$ & $0.099 *$ & -0.029 & 1.780 & $-0.128 *$ & $-0.415^{* *}$ & $3.240^{* * *}$ & $0.166^{*}$ & $0.330 * *$ & 1.220 & $49.670 *$ & $8.230 *$ \\
\hline Other SSA $\left(K_{O}=26\right)$ & $0.008 * * *$ & 0.017 & 0.040 & -0.026 & -0.089 & 0.500 & $0.064 *$ & -0.009 & 1.040 & $14.740 *$ & 0.340 \\
\hline All SSA countries $(K=40)$ & $0.067^{*}$ & -0.043 & $8.590 *$ & $-0.055 *$ & $-0.214^{*}$ & $6.010^{* *}$ & $0.112 *$ & $0.124^{* *}$ & 0.040 & $96.710 *$ & 11.270 * \\
\hline \multicolumn{12}{|l|}{ POLS_DK estimator } \\
\hline CFA Franc zone $(\mathrm{N}=14)$ & $0.102 * * *$ & 0.012 & 0.810 & -0.157 * & -0.203 & 0.090 & $0.173 *$ & 0.264 & 0.330 & $16.130 *$ & $2.750 * * *$ \\
\hline WAEMU $\left(K_{W}=08\right)$ & $0.101^{* * *}$ & 0.027 & 0.390 & $-0.142 * *$ & -0.062 & 0.170 & $0.167^{*}$ & 0.165 & 0.000 & $13.510 *$ & 0.410 \\
\hline CEMAC $\left(K_{C}=06\right)$ & 0.101 & -0.010 & 1.270 & $-0.169 *$ & $-0.359 * *$ & 1.530 & $0.177 *$ & $0.366^{* *}$ & 1.510 & $14.870 *$ & $7.400 *$ \\
\hline Other SSA $\left(K_{O}=26\right)$ & $0.101 *$ & -0.155 & $5.580 * *$ & -0.045 & 0.062 & 0.50 & $0.291 *$ & 0.037 & 2.530 & $20.750 *$ & 0.010 \\
\hline All SSA countries $(K=40)$ & $0.226^{*}$ & 0.041 & $4.370^{* *}$ & $-0.060 * * *$ & 0.002 & 0.320 & $0.269 *$ & 0.170 & 0.830 & $34.160 *$ & 0.600 \\
\hline
\end{tabular}

Note: see Table 11.

Table 14. Panel ERPT Estimation under High versus Low Inflation Levels.

\begin{tabular}{|c|c|c|c|c|c|c|c|c|c|c|}
\hline \multirow[b]{3}{*}{ Country } & \multicolumn{6}{|c|}{ Long-Term Relationship } & \multicolumn{4}{|c|}{ Short-Term Relationship } \\
\hline & \multirow[b]{2}{*}{$\begin{array}{c}\text { Asym. } \\
\text { Adjust. } \\
\text { Speed, } \beta_{i}\end{array}$} & \multirow[b]{2}{*}{$\begin{array}{c}\text { Sym. } \\
\text { Adjust. } \\
\text { Speed, } \Phi_{i}\end{array}$} & \multicolumn{3}{|c|}{ Long-Term ERPT } & \multirow[b]{2}{*}{$\begin{array}{c}\text { Wald Test } \\
\text { Long-Term } \\
H_{0}: \Omega^{-}=\Omega^{+}\end{array}$} & \multicolumn{3}{|c|}{ Cumulative ERPT } & \multirow[b]{2}{*}{$\begin{array}{c}\text { Wald Test } \\
\text { Cumulative } \\
H_{0}: \sum_{k}^{s} \theta_{i, k}^{-}=\sum_{k}^{t} \theta_{i,}^{+}\end{array}$} \\
\hline & & & $\begin{array}{c}\text { Appr(-): } \\
\Omega_{i}^{-}\end{array}$ & $\begin{array}{c}\text { Depr(+): } \\
\Omega_{i}^{+}\end{array}$ & $\begin{array}{c}\mathbf{L T}_{\text {ERPT }} \\
\Omega_{i}\end{array}$ & & $\begin{array}{c}\text { Appr(-) } \\
\sum_{k}^{s} \theta_{i, k}^{-}\end{array}$ & $\begin{array}{c}\text { Depr(+) } \\
\sum_{k}^{t} \theta_{i, k}^{+}\end{array}$ & $\begin{array}{c}\mathrm{ST}_{\mathrm{ERPT}} \\
\sum_{k}^{t} \boldsymbol{\theta}_{i, k}\end{array}$ & \\
\hline \multicolumn{11}{|l|}{ FGLS estimator } \\
\hline High inflation $(K=13)$ & $-0.029 *$ & $-0.029 *$ & $0.402 *$ & $0.157^{*}$ & 0.025 & $5.420 * *$ & -0.037 & $0.052 *$ & $0.045^{*}$ & $5.950 * *$ \\
\hline Hyperinflation $(\mathrm{K}=7)$ & -0.031 * & $-0.032 *$ & $0.395^{* *}$ & $0.166^{*}$ & $0.079 *$ & 1.970 & -0.020 & $0.061 *$ & $0.059 *$ & $2.820 * * *$ \\
\hline Low inflation $(\mathrm{K}=27)$ & $-0.016^{*}$ & $-0.014^{*}$ & $0.546^{*}$ & $0.351 *$ & $-0.057^{* *}$ & $4.590 * *$ & $-0.064^{*}$ & $0.106^{*}$ & $0.042 *$ & $119.200 *$ \\
\hline \multicolumn{11}{|l|}{ POLS_DK estimator } \\
\hline High inflation $(K=13)$ & $-0.033 *$ & $-0.032 *$ & $0.293 * *$ & $0.119 *$ & 0.031 & $3.130 * *$ & 0.0002 & $0.171 *$ & $0.166^{*}$ & $4.210 * *$ \\
\hline Hyperinflation $(K=7)$ & -0.034 * & $-0.034 *$ & $0.442 *$ & $0.152 *$ & $0.091 *$ & 4.770 ** & 0.069 & $0.185^{*}$ & $0.185^{*}$ & 1.060 \\
\hline Low inflation $(\mathrm{K}=27)$ & $-0.014^{*}$ & $-0.012 *$ & $0.771 *$ & $0.496^{*}$ & -0.051 & 2.270 & $-0.095^{* *}$ & $0.145^{* *}$ & 0.079 & $7.710^{*}$ \\
\hline
\end{tabular}

Note: $\beta_{i}$ and $\Phi_{i}$ represent the speed of adjustment in the nonlinear model (2) and the linear model (5), respectively. FGLS stands for the feasible generalized least squares; POLS DK denotes the pooled OLS (Ordinary Least Squares) estimator using Driscoll-Kraay standard errors. The statistics of Wald tests are chi-squared values. Hyperinflation indicates 7 SSA countries with average inflation level above 3.5\%. High inflation denotes 13 SSA countries where the inflation level is above the average (2.33\%) for the entire SSA region while Low inflation regroups the remaining 27 SSA countries with average inflation level below $2.33 \%$. The symbols $* * * * *$ and * show the significance at $10 \%, 5 \%$ and $1 \%$ levels respectively. Authors' calculations using Stata 14 


\section{Conclusions}

This paper investigates the relationship between the exchange rate movements and the consumer price index in 40 sub-Saharan African (SSA) countries from Q1 1990 to Q4 2017 by employing the nonlinear autoregressive distributed lag (NARDL) approach as well as dynamic panel estimators under cross-sectional dependence analysis. First, the results suggest that there was an asymmetrical exchange rate pass-through (ERPT) in most of the SSA countries generally in the short term, except for the CFA franc subregion (WAEMU and CEMAC subregions), where asymmetry occurred in both the short and long term. Second, the hypothesis of zero pass-through did not hold in many SSA countries, nor did the hypothesis of complete ERPT in the short and long term. Thus, consumer prices reacted more strongly to local currency depreciations than appreciations in the short-term, especially in the CFA Franc zone, which is in line with the result of International Monetary Fund (2012) and the capacity constraint model of Knetter (1994), suggesting the existence of trade barriers in the SSA region. This reflects downward price rigidities and weak market competition in many SSA countries. Indeed, the downward price rigidity arises from the fact that foreign producers may not be able to contain huge demand emanating from an appreciation of the importer's currency. Thus, they tend to absorb appreciations by increasing their markup and keeping prices fixed. This situation occurs in cases where producers or domestic firms are bound by quantity constraints such as quotas, trade restrictions, or anything that prevents them from expanding their production, a situation that leads to a weak competitive market in which foreign producers can only widen their profit margins rather than increasing sales. In this scenario, the pass-through will be greater during the depreciation of the importer's currency. Third, we found that the exchange rate pass-through into prices has not declined after the 1990s, especially over the long term in the CFA franc zone. It was higher in the CFA franc zone (fixed exchange rate regime) with low price levels (1.04\%) compared with the other SSA countries (floating exchange rate regime) associated with a high average inflation level (3.13\%) when the analysis allowed for cross-sectional dependence between panel units. The ERPT coefficients differed between the SSA countries and the developing and emerging Asian countries. The ERPT in the SSA countries was lower than that in emerging Asian countries in the long-term (see Kassi et al. 2018).

This evidence contrasts Taylor (2000) hypothesis and the results of Akofio-Sowah (2009) in the SSA region, revealing that neglecting the asymmetry assumption and cross-sectional dependence in such an analysis of ERPT may lead to biased conclusions. Nevertheless, we support the idea of Goldberg and Campa (2010) that the increasing share of imported inputs since the 1990s in local production processes contributes to extending the sensibility of consumer prices to exchange rate changes.

Fourth, we found an asymmetrical ERPT with regard to the size of the exchange rate. The ERPT was greater during large depreciations of the local currency than after large appreciations, whereas the ERPT for small appreciations was higher than that for small depreciations. Our findings show a lack of credibility of monetary policy in the SSA region, since inflation expectations are not well anchored on a target, reflecting little transparency in monetary policy, which may impede a declining exchange rate pass-through into consumer prices. Hence, our contribution to the ERPT literature in the SSA region is threefold: First, we extended the analysis to 40 SSA countries by using both per-country and dynamic panel analysis, allowing for asymmetry. Second, we considered the cross-sectional dependence analysis between countries in the estimation of ERPT. Third, our analysis took into account nonlinearity by examining the pass-through with regard to the size of the exchange rate change. Our findings also raise concerns about probably speculative behavior from producers with great market power to make use of the asymmetric exchange rate pass-through in order to profit from weak competitive market structures in the SSA region. Such behavior can reduce consumer welfare in the destination market following the response of local prices and thereby may hinder the monetary policy of inflation targeting and export competitiveness.

Finally, the ERPT was low under the floating exchange rate regime relative to the fixed regime. Hence, the SSA countries should not be in fear of floating, as they can benefit from trade liberalization. Therefore, the policy implication is to take into account these asymmetrical effects 
of exchange rates on consumer prices when determining the monetary policy rules, to promote trade liberalization and enhance macroeconomic policies for more competitive market structures in sub-Saharan African countries.

Author Contributions: All authors contributed equally to all aspects of this research.

Funding: This research has not received external funding.

Conflicts of Interest: The authors declare no conflict of interest.

\section{Appendix A}

This part discusses the four tests used in the cross-sectional dependence analysis mentioned in Section 3.3: those developed by Friedman (1937), Breusch and Adrian (1980), Frees (1995), and Pesaran (2004).

The Friedman (1937) test is nonparametric and based on Spearman's rank correlation coefficient. Friedman's test statistic is computed as follows:

$$
R_{\text {ave }}=\frac{2}{\mathrm{~K}(\mathrm{~K}-1)} \sum_{i=1}^{K-1} \sum_{j=i+1}^{K} \hat{r_{i j}}
$$

where $r_{i j}$ is the Spearman correlation coefficient.

Breusch and Adrian (1980) proposed an alternative statistic based on the Lagrange multiplier, which is suitable for large time periods $(\mathrm{T}>\mathrm{K})$ and relatively small cross-section dimension $\mathrm{K}$ and tests the null hypothesis of no cross-sectionally correlated errors:

$$
C D_{L M}=T \sum_{i}^{K-1} \sum_{j=i+1}^{K} \hat{\rho}_{i j}^{2}
$$

where $\hat{\rho}_{i j}$ is the estimated pairwise correlation of the residuals.

In addition, Frees (1995) provided another powerful cross-sectional dependence test that can deal with the false hypothesis in the case of many disturbances that are cross-sectionally correlated. Frees's statistic contains the coefficients of the squared rank correlation and is calculated as follows:

$$
F T=K\left\{R_{a v e}^{2}-(T-1)^{-1}\right\} \stackrel{d}{\rightarrow} Q \text { distribution and } R_{a v e}^{2}=\frac{2}{\mathrm{~K}(\mathrm{~K}-1)} \sum_{i=1}^{K-1} \sum_{j=i+1}^{K} r_{i j}^{2}
$$

Pesaran (2004) suggested a modified version of Breusch and Adrian (1980) statistic and tested the null hypothesis of zero cross-section correlation among residuals when $\mathrm{T}<\mathrm{K}$. Pesaran's CD statistic is computed as $C D=\sqrt{\frac{2 T}{\mathrm{~K}(\mathrm{~K}-1)}}\left(\sum_{i=1}^{K-1} \sum_{j=i+1}^{K} \sqrt{T_{i j}} \hat{\rho}_{i j}\right)$ for unbalanced panels, $C D \rightarrow K(0,1)$ for large $\mathrm{T}$ and $K \rightarrow \infty$. Thus, the Friedman (1937), Pesaran (2004), and Frees (1995) tests are useful cross-sectional dependence tests when $\mathrm{T}<\mathrm{K}$, while the Breusch and Adrian (1980) LM test is suitable for $\mathrm{T}>\mathrm{K}$. 


\section{WAEMU}
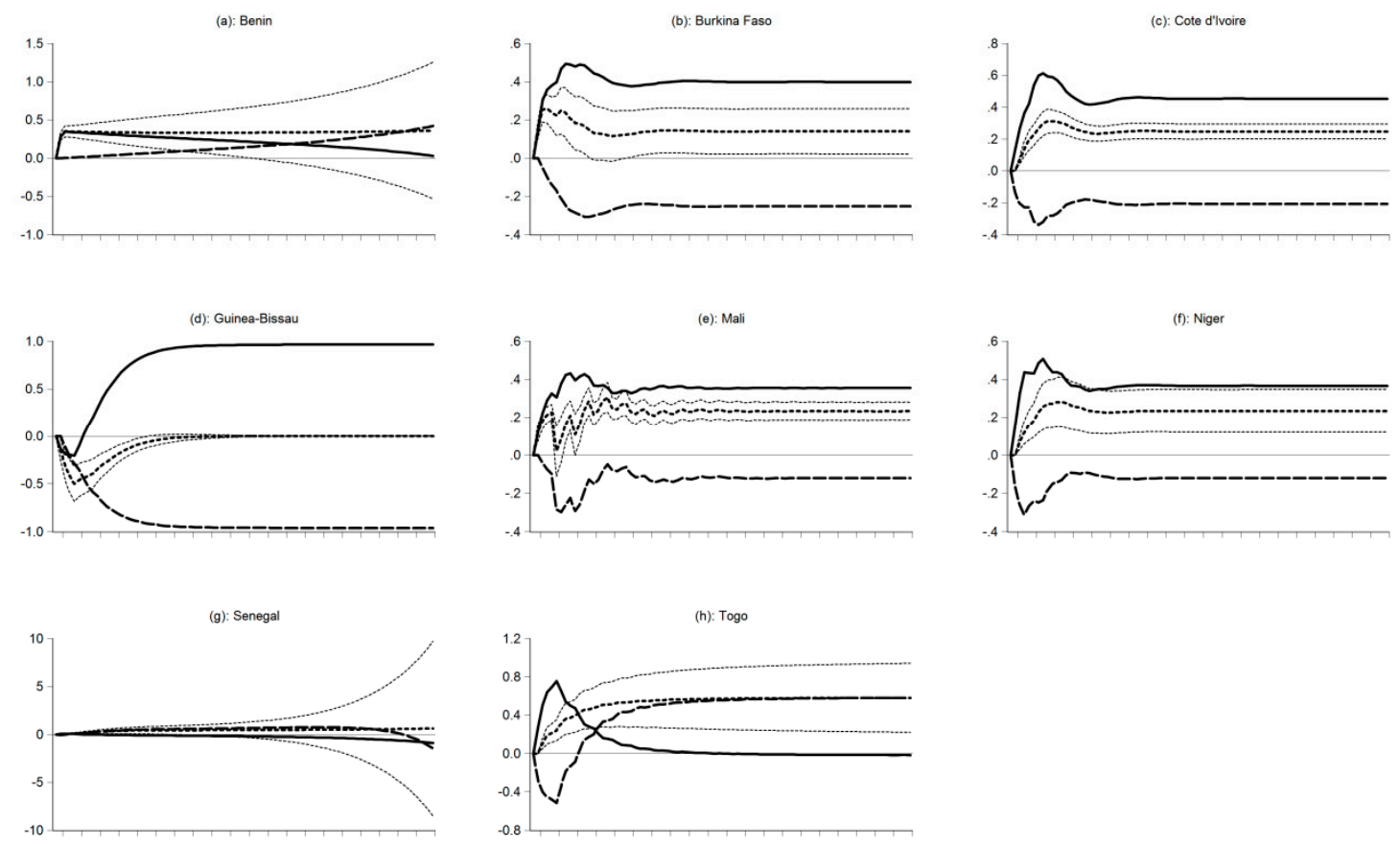

\section{CEMAC}
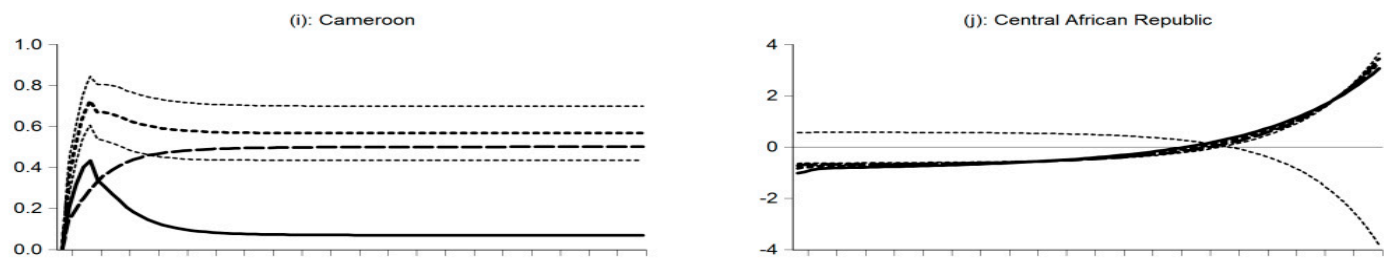

(k): Chad

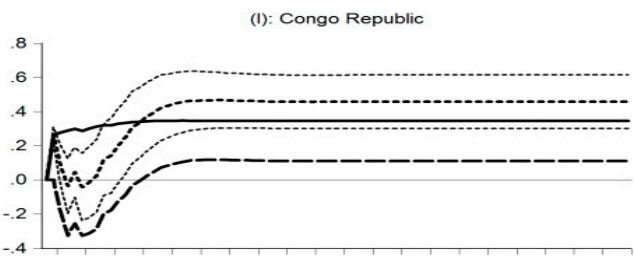

(m): Equatorial Guinea

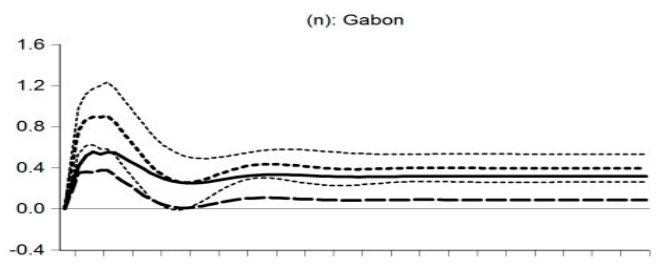

Figure A1. Cumulative dynamic multipliers for CFA franc zone. Note: The dynamic multipliers were generated by the authors using Eviews 9. The long-dashed (solid) line depicts the effect of $1 \%$ appreciation (depreciation) of the local currency, i.e., negative and positive changes of exchange rate on consumer prices, respectively. The asymmetry line, or the difference between depreciations and appreciations, is described by the short-dashed line. The horizontal and vertical lines show the time horizon and the magnitude of the pass-through, respectively. 

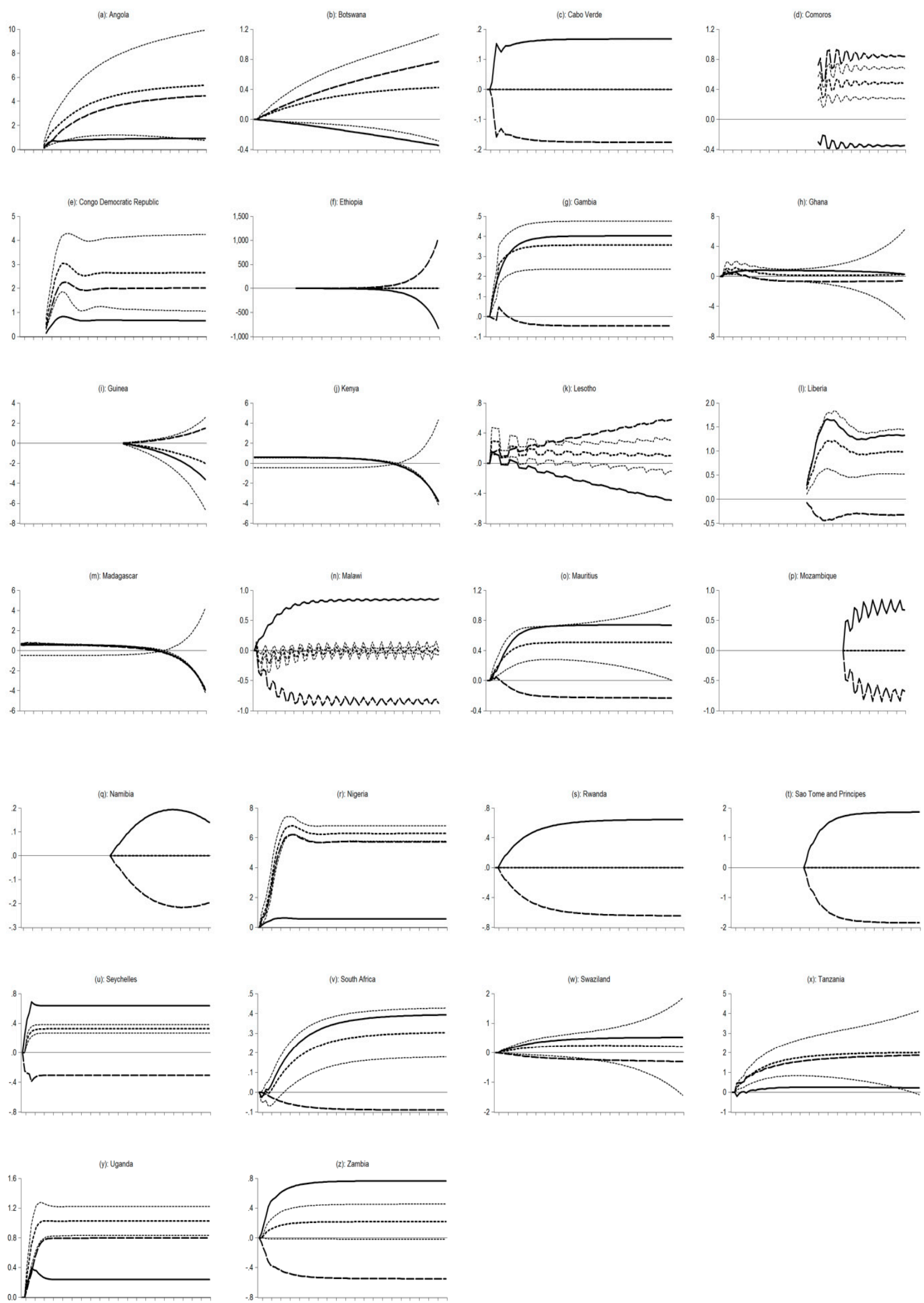

Figure A2. Cumulative dynamic multipliers for the other SSA countries. Note: See note on Figure A1. 


\section{Appendix B}

Appendix B.1

Table A1. The critical values of Narayan (2005).

\begin{tabular}{|c|c|c|c|c|c|c|c|c|c|c|c|}
\hline & & Obs. & 40 & 45 & 50 & 55 & 60 & 65 & 70 & 75 & 80 \\
\hline \multirow{4}{*}{$1 \%$} & \multirow{2}{*}{$\mathrm{k}=4$} & $\mathrm{I}(0)$ & 4.428 & 4.394 & 4.306 & 4.244 & 4.176 & 4.188 & 4.098 & 4.168 & 4.096 \\
\hline & & $\mathrm{I}(1)$ & 6.250 & 5.914 & 5.874 & 5.726 & 5.676 & 5.694 & 5.570 & 5.548 & 5.512 \\
\hline & \multirow[b]{2}{*}{$\mathrm{k}=5$} & $\mathrm{I}(0)$ & 4.045 & 4.030 & 3.955 & 3.928 & 3.783 & 3.783 & 3.747 & 3.772 & 3.725 \\
\hline & & $\mathrm{I}(1)$ & 5.898 & 5.598 & 5.583 & 5.408 & 5.338 & 5.300 & 5.285 & 5.213 & 5.163 \\
\hline \multirow{4}{*}{$5 \%$} & \multirow[b]{2}{*}{$\mathrm{k}=4$} & $\mathrm{I}(0)$ & 3.202 & 3.178 & 3.136 & 3.068 & 3.062 & 3.068 & 3.022 & 3.042 & 3.010 \\
\hline & & $\mathrm{I}(1)$ & 4.544 & 4.450 & 4.416 & 4.334 & 4.314 & 4.274 & 4.256 & 4.244 & 4.216 \\
\hline & \multirow{2}{*}{$\mathrm{k}=5$} & $\mathrm{I}(0)$ & 2.962 & 2.922 & 2.900 & 2.848 & 2.817 & 2.835 & 2.788 & 2.802 & 2.787 \\
\hline & & $\mathrm{I}(1)$ & 4.338 & 4.268 & 4.218 & 4.160 & 4.097 & 4.090 & 4.073 & 4.065 & 4.015 \\
\hline \multirow{4}{*}{$10 \%$} & \multirow[b]{2}{*}{$\mathrm{k}=4$} & $\mathrm{I}(0)$ & 2.660 & 2.638 & 2.614 & 2.578 & 2.568 & 2.574 & 2.552 & 2.558 & 2.548 \\
\hline & & $\mathrm{I}(1)$ & 3.838 & 3.772 & 3.746 & 3.710 & 3.712 & 3.682 & 3.648 & 3.654 & 3.644 \\
\hline & \multirow{2}{*}{$\mathrm{k}=5$} & $\mathrm{I}(0)$ & 2.483 & 2.458 & 2.435 & 2.393 & 2.385 & 2.397 & 2.363 & 2.380 & 2.355 \\
\hline & & $\mathrm{I}(1)$ & 3.708 & 3.647 & 3.600 & 3.583 & 3.565 & 3.543 & 3.510 & 3.515 & 3.500 \\
\hline
\end{tabular}

Note: The critical values have been extracted from Narayan (2005). Obs. denotes the number of observations. $\mathrm{k}$ is the number of independent variables. I(0) and I(1) represent the order of integration.

Appendix B.2

Table A2. Classification of the SSA Countries according to the Inflation levels.

\begin{tabular}{|c|c|c|}
\hline $\begin{array}{l}\text { Countries with High Inflation }(K=13 \text { ) } \\
\text { (Average Inflation Above } 2.33 \% \text { ) }\end{array}$ & $\begin{array}{l}\text { Countries with Hyperinflation }(K=7) \\
\text { (Average Inflation Above 3.5\%) }\end{array}$ & $\begin{array}{l}\text { Countries with Low Inflation }(K=27) \\
\text { (Average-Inflation Below } 2.33 \%)\end{array}$ \\
\hline $\begin{array}{l}\text { Guinea-Bissau, Angola, Congo } \\
\text { Democratic Republic, Ghana, Guinea, } \\
\text { Kenya, Liberia, Madagascar, Malawi, } \\
\text { Nigeria, Sao Tome and Principe, } \\
\text { Tanzania and Zambia }\end{array}$ & $\begin{array}{l}\text { Angola, Congo Democratic Republic, } \\
\text { Ghana, Guinea, Malawi, } \\
\text { Nigeria and Zambia }\end{array}$ & $\begin{array}{l}\text { Benin, Burkina Faso, Cote d'Ivoire, Mali, } \\
\text { Niger, Senegal, Togo, Cameroon, } \\
\text { Central African Republic, Chad, Congo } \\
\text { Republic, Equatorial Guinea, Gabon, } \\
\text { Botswana, Cabo Verde, Comoros, } \\
\text { Ethiopia, Gambia, Lesotho, Mauritius, } \\
\text { Mozambique, Namibia, Rwanda, } \\
\text { Seychelles, South Africa, } \\
\text { Swaziland and Uganda. }\end{array}$ \\
\hline
\end{tabular}

Note: The value $2.33 \%$ represent the average inflation of the entire SSA region as indicated in Table 2 . However, the value $3.5 \%$ is an arbitrary value showing that the countries have higher inflation levels far beyond the average inflation $(2.33 \%)$ compared to the others but also in accordance to the recent inflation trends in these countries over our sample period. $\mathrm{K}$ denotes the number of countries in each group.

\section{References}

Akofio-Sowah, Naa Anyeley. 2009. Is There a Link Between Exchange Rate Pass-Through and the Monetary Regime: Evidence from Sub-Saharan Africa and Latin America. International Advances in Economic Research 15: 296-309. [CrossRef]

An, Lian, and Jian Wang. 2011. Exchange Rate Pass-Through: Evidence Based on Vector Autoregression with Sign Restrictions. Open Economies Review 23: 359-80. [CrossRef]

Bada, Abiodun, Ajibola Olufemi, Inuwa Tata, Idowu Peters, Sani Bawa, Anigwe Onwubiko, and Udoko Onyowo. 2016. Exchange rate pass-through to inflation in Nigeria. CBN Journal of Applied Statistics 7: 49-70.

Baharumshah, Ahmad Zubaidi, Abdalla Sirag, and Siew-Voon Soon. 2017. Asymmetric exchange rate pass-through in an emerging market economy: The case of Mexico. Research in International Business and Finance 41: 247-59. [CrossRef]

Bahmani-Oskooee, Mohsen, and Amirhossein Mohammadian. 2017. Asymmetry Effects of Exchange Rate Changes on Domestic Production in Emerging Countries. Emerging Markets Finance and Trade 54: 1442-59. [CrossRef] 
Banerjee, Anindya, Juan Dolado, and Ricardo Mestre. 1998. Error-correction Mechanism Tests for Cointegration in a Single-equation Framework. Journal of Time Series Analysis 19: 267-83. [CrossRef]

Beck, Nathaniel, and Jonathan N. Katz. 1995. What to Do (and Not to Do) with Time-Series Cross-Section Data. American Political Science Review 89: 634-47. [CrossRef]

Ben Cheikh, Nidhaleddine. 2012. Asymmetric Exchange Rate Pass-Through in the Euro Area: New Evidence from Smooth Transition Models. Economics: The Open-Access, Open-Assessment E-Journal 6: 1. [CrossRef]

Berner, Eike. 2010. Exchange rate pass-through: New evidence from German micro data. International Economics 124: 75-100. [CrossRef]

Bhundia, Ashok. 2002. An Empirical Investigation of Exchange Rate Pass-Through in South Africa. IMF Working Papers. Washington, DC: International Monetary Fund. [CrossRef]

Breusch, Trevor, and Pagan Adrian. 1980. The Lagrange Multiplier Test and its Applications to Model Specification in Econometrics. The Review of Economic Studies. [CrossRef]

Brun-Aguerre, Raphael, Ana-Maria Fuertes, and Kate Phylaktis. 2012. Exchange rate pass-through into import prices revisited: What drives it? Journal of International Money and Finance 31: 818-44. [CrossRef]

Brun-Aguerre, Raphael, Ana-Maria Fuertes, and Matthew Greenwood-Nimmo. 2016. Heads I win; tails you lose: Asymmetry in exchange rate pass-through into import prices. Journal of the Royal Statistical Society: Series A (Statistics in Society) 180: 587-612. [CrossRef]

Bui, Minh Tam. 2018. Causality in Vietnam's Parallel Exchange Rate System during 2005-2011: Policy Implications for Macroeconomic Stability. Economies 6: 68. [CrossRef]

Bussière, Matthieu, Simona Delle Chiaie, and Tuomas Peltonen. 2014. Exchange Rate Pass-Through in the Global Economy: The Role of Emerging Market Economies. IMF Economic Review 62: 146-78. [CrossRef]

Campa, Jose Manuel, and Linda Goldberg. 2002. Exchange Rate Pass-Through into Import Prices: A Macro or Micro Phenomenon? SSRN Electronic Journal. [CrossRef]

Campa, Jose Manuel, and Linda Goldberg. 2005. Exchange Rate Pass-Through into Import Prices. Review of Economics and Statistics 87: 679-90. [CrossRef]

Choudhri, Ehsan U., and Dalia S. Hakura. 2015. The exchange rate pass-through to import and export prices: The role of nominal rigidities and currency choice. Journal of International Money and Finance 51: 1-25. [CrossRef]

Choudhri, Ehsan U., Hamid Faruqee, and Dalia S. Hakura. 2005. Explaining the exchange rate pass-through in different prices. Journal of International Economics 65: 349-74. [CrossRef]

Conrad, Daren, and Jaymieon Jagessar. 2018. Real Exchange Rate Misalignment and Economic Growth: The Case of Trinidad and Tobago. Economies 6: 52. [CrossRef]

Delatte, Anne-Laure, and Antonia Lòpez-Villavicencio. 2012. Asymmetric exchange rate pass-through: Evidence from major countries. Journal of Macroeconomics 34: 833-44. [CrossRef]

Dickey, David, and Wayne Fuller. 1981. Likelihood Ratio Statistics for Autoregressive Time Series with a Unit Root. Econometrica. [CrossRef]

Dornbusch, Rudiger. 1985. Exchange Rates and Prices. NBER Working Paper No. 1769. Cambridge: National Bureau of Economic Research. [CrossRef]

Driscoll, John, and Aart Kraay. 1998. Consistent Covariance Matrix Estimation with Spatially Dependent Panel Data. Review of Economics and Statistics 80: 549-60. [CrossRef]

Frankel, Jeffrey, David Parsley, and Shang-Jin Wei. 2011. Slow Pass-through Around the World: A New Import for Developing Countries? Open Economies Review 23: 213-51. [CrossRef]

Frees, Edward W. 1995. Assessing cross-sectional correlation in panel data. Journal of Econometrics 69: $393-414$. [CrossRef]

Friedman, Milton. 1937. The Use of Ranks to Avoid the Assumption of Normality Implicit in the Analysis of Variance. Journal of the American Statistical Association. [CrossRef]

Frimpong, Siaw, and Anokye Adam. 2010. Exchange Rate Pass-Through in Ghana. International Business Research. [CrossRef]

Goldberg, Linda S., and Jose Manuel Campa. 2010. The Sensitivity of the CPI to Exchange Rates: Distribution Margins, Imported Inputs, and Trade Exposure. The Review of Economics and Statistics 2: 392-407. [CrossRef]

Goldberg, Pinelopi, and Michael Knetter. 1996. Goods Prices and Exchange Rates: What Have We Learned? Cambridge: National Bureau of Economic Research. [CrossRef] 
Hoechle, Daniel. 2007. Robust Standard Errors for Panel Regressions with Cross-Sectional Dependence. The Stata Journal 7: 281-312. [CrossRef]

International Monetary Fund. 2012. Exchange Rate Pass-Through in Sub-Saharan African Economies and its Determinants. IMF Working Papers. Washington, DC: International Monetary Fund. [CrossRef]

Jombo, Wytone, Kisu Simwaka, and Austin Chiumia. 2014. Exchange Rate Pass-Through in Malawi: Evidence from Augmented Phillips Curve and Vector Autoregressive Approaches. Standard Global Journal of Business Management 1: 34-40.

Jooste, Charl, and Yaseen Jhaveri. 2014. The Determinants of Time-Varying Exchange Rate Pass-Through in South Africa. South African Journal of Economics 82: 603-15. [CrossRef]

Karoro, Tapiwa D., Meshach J. Aziakpono, and Nicolette Cattaneo. 2009. Exchange Rate Pass-Through to Import Prices in South Africa: Is There Asymmetry? South African Journal of Economics 77: 380-98. [CrossRef]

Kassi, Diby François, Gang Sun, Ning Ding, Dilesha Nawadali Rathnayake, and Guy Roland Assamoi. 2018. Asymmetry in exchange rate pass-through to consumer prices: Evidence from emerging and developing Asian countries. Economic Analysis and Policy. [CrossRef]

Kmenta, Jan. 1986. Elements of Econometrics, 2nd ed. New York: Macmillan.

Knetter, Michael. 1994. Is export price adjustment asymmetric?: Evaluating the market share and marketing bottlenecks hypotheses. Journal of International Money and Finance 13: 55-70. [CrossRef]

Krugman, Paul. 1986. Pricing to Market when the Exchange Rate Changes. Cambridge: National Bureau of Economic Research. [CrossRef]

Kwiatkowski, Denis, Peter C. B. Phillips, Peter Schmidt, and Yongcheol Shin. 1992. Testing the null hypothesis of stationarity against the alternative of a unit root. Journal of Econometrics 54: 159-78. [CrossRef]

Maka, Evans Darnor. 2013. Asymmetric Pass-Through of Exchange Rate Changes to CPI Inflation in Ghana. International Journal of Economics, Finance and Management 2: 338-50.

Marston, Richard C. 1990. Pricing to market in Japanese manufacturing. Journal of International Economics 29: 217-36. [CrossRef]

Mwase, Nkunde. 2006. An Empirical Investigation of the Exchange Rate Pass-Through to Inflation in Tanzania. IMF Working Papers. Washington, DC: International Monetary Fund. [CrossRef]

Narayan, Paresh Kumar. 2005. The saving and investment nexus for China: Evidence from cointegration tests. Applied Economics 37: 1979-90. [CrossRef]

Newey, Whitney K., and Kenneth West. 1987. A Simple, Positive Semi-Definite, Heteroskedasticity and Autocorrelation Consistent Covariance Matrix. Econometrica 55: 703-8. [CrossRef]

Olivei, Giovanni P. 2002. Exchange rates and the prices of manufacturing products imported into the United States. New England Economic Review, 3-18.

Otani, Akira, Shigenori Shiratsuka, and Toyoichiro Shirota. 2003. The Decline in the Exchange Rate Pass-Through: Evidence from Japanese Import Prices. Institute for Monetary and Economic Studies 21: 53-81.

Ozkan, Ibrahim, and Luffi Erden. 2015. Time-varying nature and macroeconomic determinants of exchange rate pass-through. International Review of Economics \& Finance 38: 56-66. [CrossRef]

Parks, Richard W. 1967. Efficient Estimation of a System of Regression Equations when Disturbances are Both Serially and Contemporaneously Correlated. Journal of the American Statistical Association. [CrossRef]

Pesaran, Hashem M. 2004. General Diagnostic Tests for Cross Section Dependence in Panels. CESifo Working Paper Series No. 1229; IZA Discussion Paper No. 1240. Cambridge, UK: University of Cambridge.

Pesaran, Hashem M., Yongcheol Shin, and Richard J. Smith. 2001. Bounds testing approaches to the analysis of level relationships. Journal of Applied Econometrics 16: 289-326. [CrossRef]

Phillips, Peter C. B., and Pierre Perron. 1988. Testing for a Unit Root in Time Series Regression. Biometrika 75: 335-46. [CrossRef]

Pollard, Patricia S., and Cletus Coughlin. 2004. Size Matters: Asymmetric Exchange Rate Pass-Through at the Industry Level. SSRN Electronic Journal. [CrossRef]

Savoie-Chabot, Laurence, and Mikael Khan. 2015. Exchange Rate Pass-Through to Consumer Prices: Theory and Recent Evidence. Discussion Paper 2015-9. Ottawa: Bank of Canada.

Sbia Rashid, Muhammad Shahbaz, and Helmi Hamdi. 2014. A contribution of foreign direct investment, clean energy, trade openness, carbon emissions and economic growth to energy demand in UAE. Economic Modelling 36: 191-97. [CrossRef] 
Shahbaz, Muhammad, Thi Hong Van Hoang, Mantu Kumar Mahalik, and David Roubaud. 2017. Energy consumption, financial development and economic growth in India: New evidence from a nonlinear and asymmetric analysis. Energy Economics 63: 199-212. [CrossRef]

Shin, Yongcheol, Byungchul Yu, and Matthew Greenwood-Nimmo. 2014. Modelling Asymmetric Cointegration and Dynamic Multipliers in a Nonlinear ARDL Framework. In Festschrift in Honor of Peter Schmidt. New York: Springer, pp. 281-314. [CrossRef]

Takhtamanova, Yelena F. 2010. Understanding changes in exchange rate pass-through. Journal of Macroeconomics 32: 1118-30. [CrossRef]

Taylor, John Bernard. 2000. Low inflation, pass-through, and the pricing power of firms. European Economic Review 44: 1389-408. [CrossRef]

Usman, Ojonugwa, and Osama Mohammed Elsalih. 2018. Testing the Effects of Real Exchange Rate Pass-Through to Unemployment in Brazil. Economies 6: 49. [CrossRef]

Utku Özmen, Mustafa, and Fatih Akçelik. 2017. Asymmetric exchange rate and oil price pass-through in motor fuel market: A microeconometric approach. The Journal of Economic Asymmetries 15: 64-75. [CrossRef]

Ware, Roger, and Ralph Winter. 1988. Forward markets, currency options and the hedging of foreign exchange risk. Journal of International Economics 25: 291-302. [CrossRef]

Yanamandra, Venkataramana. 2015. Exchange rate changes and inflation in India: What is the extent of exchange rate pass-through to imports? Economic Analysis and Policy 47: 57-68. [CrossRef]

Yellen, Janet Louise. 2015. Inflation Dynamics and Monetary Policy. Paper presented at the Philip Gamble Memorial Lecture, University of Massachusetts, Amherst, MA, USA, September 24.

(C) 2019 by the authors. Licensee MDPI, Basel, Switzerland. This article is an open access article distributed under the terms and conditions of the Creative Commons Attribution (CC BY) license (http:/ / creativecommons.org/licenses/by/4.0/). 